Final Report on NASA Agreement No. NAG-1-2052

\title{
BOLTED DOUBLE-LAP COMPOSITE JOINTS UNDER MECHANICAL AND THERMAL LOADING
}

\author{
SUBMITTED BY \\ V. Kradinov, A. Barut, and E. Madenci \\ Department of Aerospace and Mechanical Engineering \\ The University of Arizona \\ Tucson, AZ 85721
}

\author{
SUBMITTED TO \\ Sandra P. Walker, IARC Technical Officer \\ Mail Stop 396 \\ NASA Langley Research Center \\ 100 NASA Road \\ Hampton, VA 23681-2199
}

August 2000 


\section{TABLE OF CONTENTS}

\section{Page}

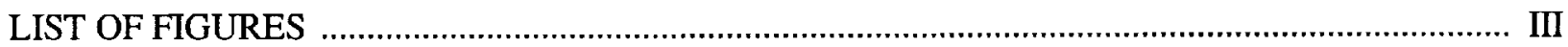

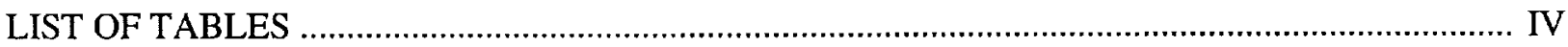

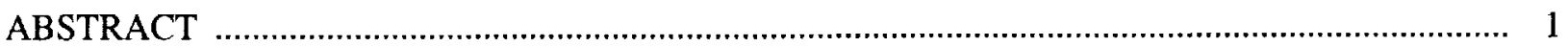

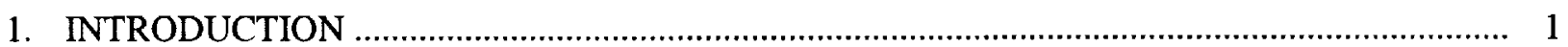

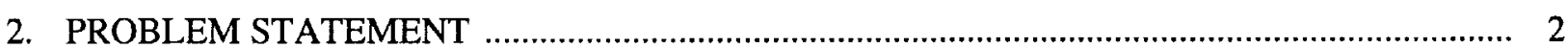

3. SOLUTION METHOD

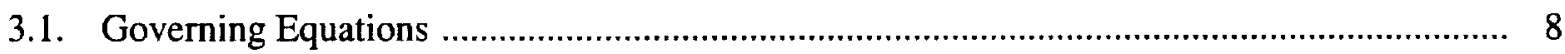

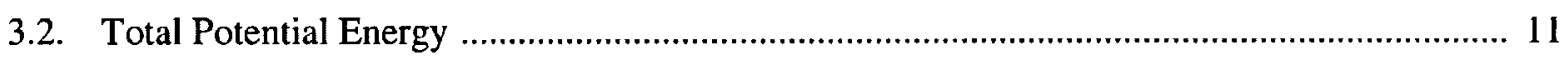

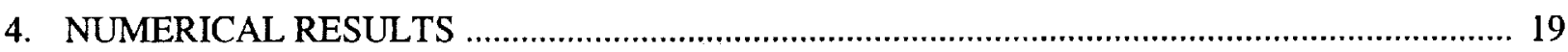

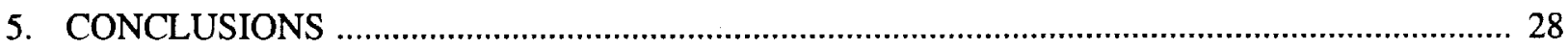

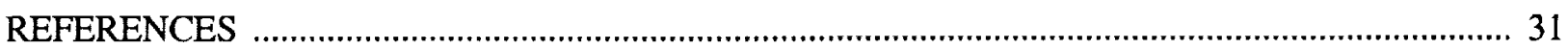

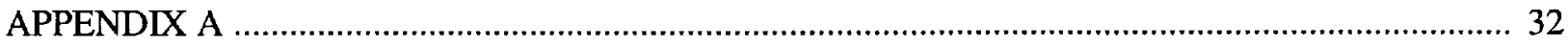

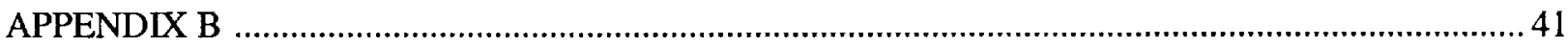

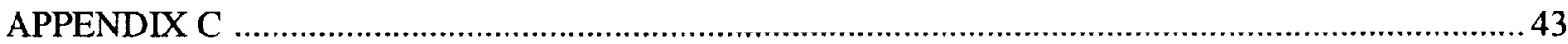

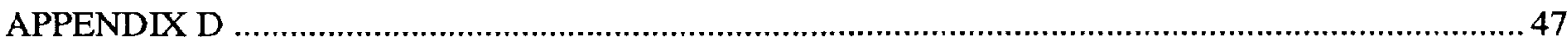




\section{LIST OF FIGURES}

Figure

1 Geometric description of single- and double-lap joints with many bolts ................................. 3

2 Position of a bolt before and after the load is executed ........................................................ 4

$3 \quad$ Position of a bolt before and after the load is executed f...................................................... 5

4 The gap between the bolt and hole boundary immediately before and after

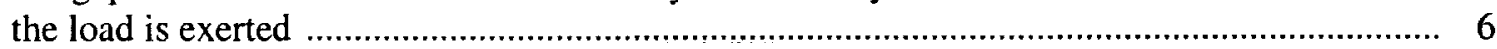

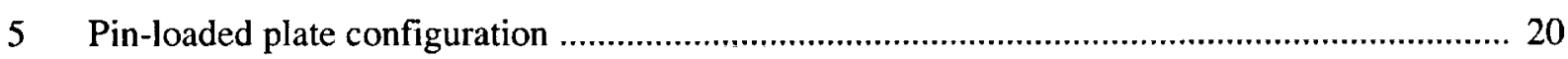

6 Comparison of normalized stress around hole for pin-loaded plate for laminate type A .......... 21

7 Comparison of normalized stress around hole for pin-loaded plate for laminate type B .......... 22

8 Comparison of normalized stress around hole for pin-loaded plate for laminate type C .......... 23

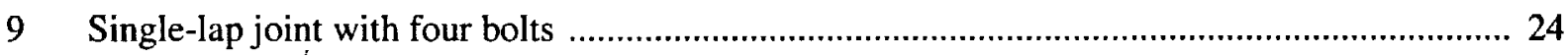

10 Steel and Aluminum plates connected with a single bolt under thermal loading ................... 24

11 Steel plates bolted to an aluminum plate with three bolts ................................................. 26

12 Stresses around bolt holes in alloy plate due to mechanical loading only .............................. 26

13 Stresses around bolt holes in alloy plate due to thermal loading only ..................................... 27

14 Stresses around bolt holes in alloy plate due to combined thermal-mechanical loading ........... 27

15 Steel plates bolted to a composite laminate with seven bolts ................................................ 29

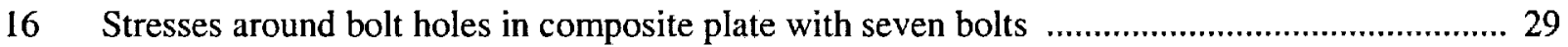

Al A close view of the section in the vicinity of a bolt, and the discretization of the bolt into Timoshenko beam elements .......................................................................................... 32

D1 The behavior of radial stress near the start point of a contact region ..................................... 48

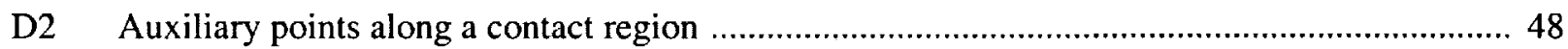




\section{LIST OF TABLES}

Table

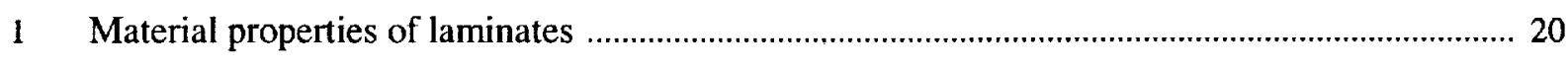

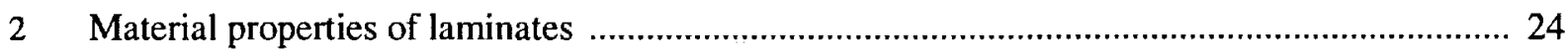

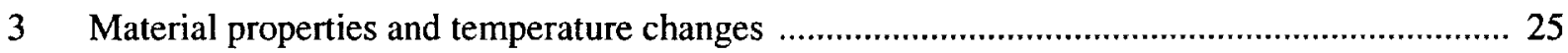

4 Bolt load distribution in a double-lap joint of steel plates and an aluminum plate with three bolts

5 Bold load distribution in a double-lap joint of steel plates and a composite laminate with seven bolts 


\begin{abstract}
This study concerns the determination of the contact stresses and contact region around bolt holes and the bolt load distribution in single- and double-lap joints of composite laminates with arbitrarily located bolts under general mechanical loading conditions and uniform temperature change. The unknown contact stress distribution and contact region between the bolt and laminates and the interaction among the bolts require the bolt load distribution, as well as the contact stresses, to be as part of the solution. The present method is based on the complex potential theory and the variational formulation in order to account for bolt stiffness, bolt-hole clearance, and finite geometry of the composite laminates.
\end{abstract}

\title{
1. INTRODUCTION
}

Bolted joints provide the primary means for transferring load among composite components in the construction of aircraft and space structures. The stress state in a bolted joint is dependent primarily on the dimensions of the planar geometry, loading conditions, degree of material anisotropy, bolt-hole clearance, bolt flexibility, and friction between the laminates. Also, aircraft and space vehicles traveling at supersonic and hypersonic speeds can experience high temperature excursions. The influence of thermal expansions can be significant and may differ significantly among the materials for the bolts and laminates. As a result, high thermal stresses may develop as the temperature increases and may alter the bolt load distribution. Therefore, accurate determination of the stresses in bolted laminates under both mechanical and thermal loading is essential for reliable strength evaluation and failure prediction.

A considerable amount of work on the behavior of composite joints with a single bolt exists in the literature. These studies investigated the stress distribution around a pin-loaded hole in laminated composites based on either finite element analysis or analytical methods. Since the contact stress distribution and the contact region are not known a priori, a majority of the models did not directly impose the boundary conditions appropriate for modeling the contact and non-contact regions between the bolt and the boundary of the hole. These models usually assumed a cosinusoidal bearing stress distribution or zero radial displacements over the contact region of the hole boundary. In the case of multi-bolt joints, the commonly accepted approach is to first determine the load distribution among the bolts in order to identify the critical (most highly loaded) bolt for a subsequent single-bolt analysis for local stress distribution. However, this type of analysis disregards the interaction among the bolts located in close proximity to each other. In order to eliminate these shortcomings, Madenci et al. (1998) developed a method for single-lap joints based on the boundary collocation technique. Their method determines the contact stresses and contact region, as well as the bolt load distribution, as part of the 
solution procedure. However, this method fails to provide converged solutions consistently depending on the number of bolts and their location in relation to each other or to the free boundaries. A detailed validation and demonstration of their approach, as well as an extensive review of previous analyses, were reported in detail by Madenci et al. (1997).

In the literature, there are essentially no direct analyses of double-lap bolted joints for solid laminates under general loading conditions and appropriate boundary conditions arising from contact phenomenon. Madenci et al. (1999) extended their boundary collocation technique for single-lap joints to consider double-lap joints and thermal loading. This method provided converged results for particular configurations, but also suffered from consistent convergence arising from the explicit partitioning of the domain.

Xiong and Poon (1994) introduced an analytical approach utilizing a variational formulation in conjunction with the complex potential theory to single- and double-lap joints with many bolts. Their approach considers each laminate of the joint separately. The coupling of the laminates is achieved through bolt displacements, which are permitted only in the direction of loading. In their two-stage analysis, the first stage provides the local deformation along the hole boundaries of one of the laminates subjected to the external boundary conditions and the prescribed cosinusoidal bearing stress representing the bolt load at each hole boundary. The local deformations and the bolt deflections are imposed as displacement constraints in the subsequent second stage to determine the contact stresses (bolt loads) and the contact region in the second laminate. Subsequently, these fastener loads are imposed as prescribed cosinusoidal bearing stress for the first stage of the analysis, and the iterative process continues until the constraint conditions are satisfied.

This study presents an analysis method for determining the bolt load distribution in single- and double-lap joints while accounting for the contact phenomenon and the interaction among the bolts explicitly under bearing and by-pass loading with or without thermal loading. It is an extension of the analysis introduced by Xiong and Poon (1994) and eliminates the requirement of a two-stage analysis and the associated iterative process. The resulting equations are solved in a coupled manner, leading to the contact stresses, contact region, and bolt load distribution.

\section{PROBLEM STATEMENT}

The geometry of a bolted single- and double-lap joint with composite laminates is described in Fig. 1 . The joint can be subjected to a combination of bearing, by-pass and shear loads, and a uniform temperature change. Each laminate of the single- and double-lap joints, joined with $L$ number of bolts, is subjected to traction components, $\bar{t}_{\alpha}^{(k)}(\alpha=x, y)$ along the $\bar{\Gamma}^{(k)}$ segment of the external boundary of each region 


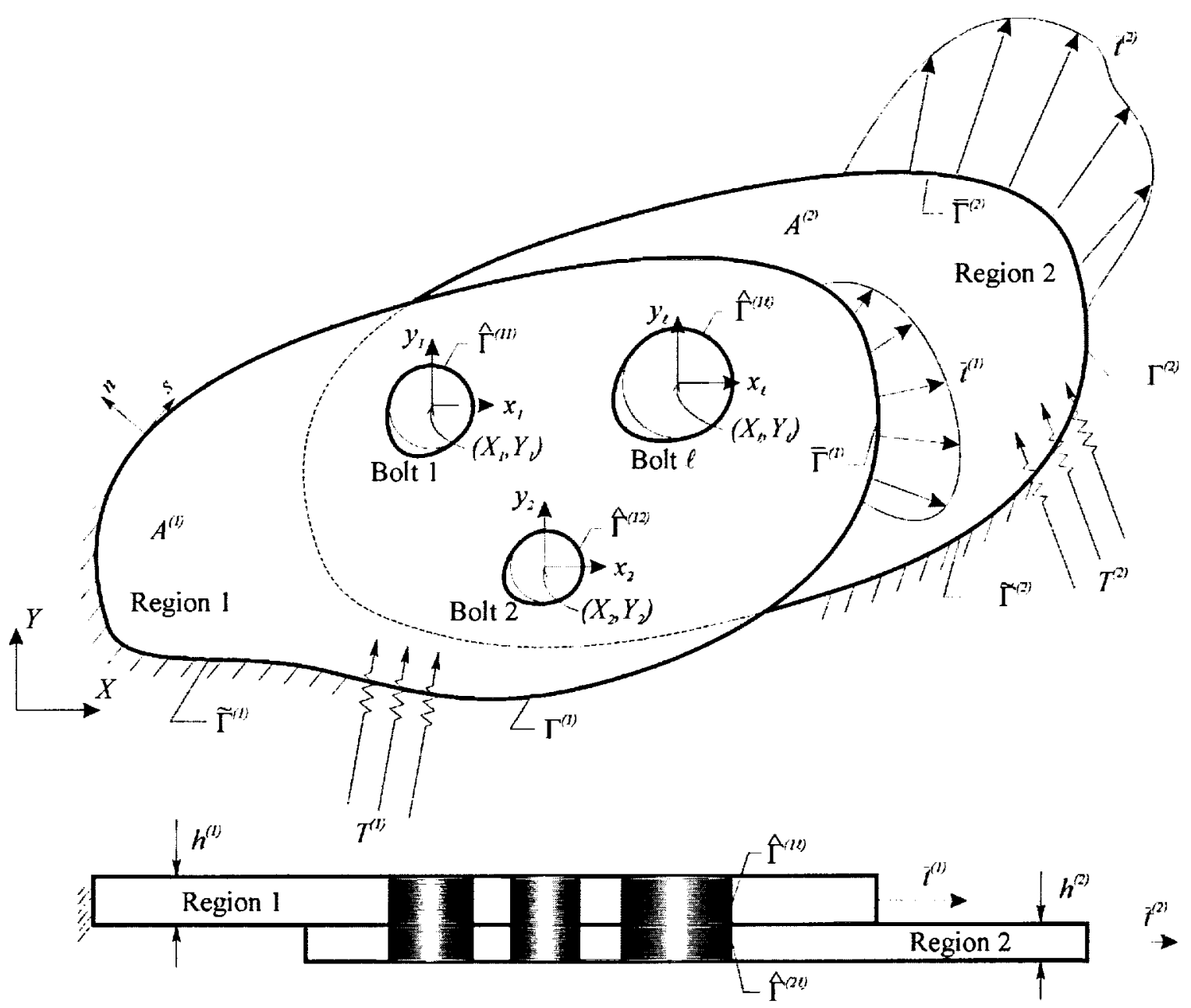

Single-lap joint

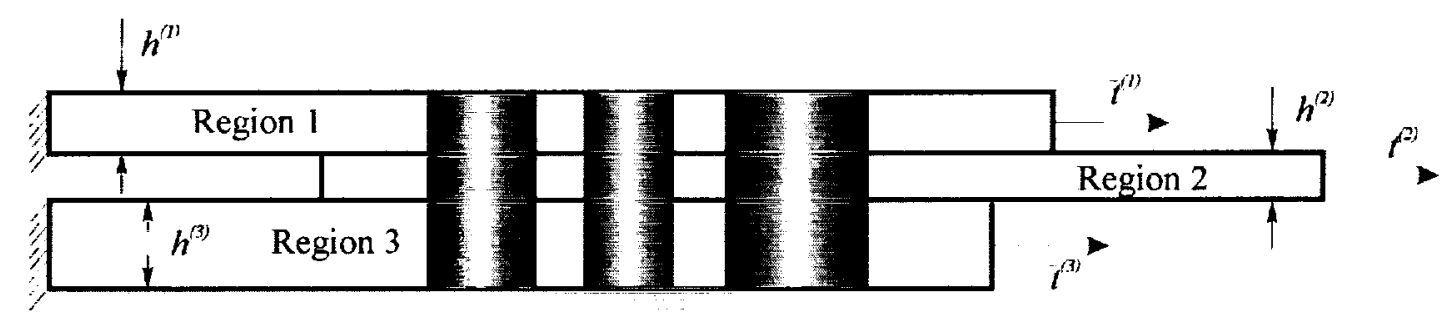

Double-lap joint

Fig. 1. Geometric description of single- and double-lap joints with many bolts. 
denoted by $\Gamma^{(k)}$. The section of the external boundary subjected to displacement constraints, $\tilde{u}_{\rho}^{(k)}$ $(\rho=n, s)$, is denoted by $\tilde{\Gamma}^{(k)}$. The subscripts " $n$ " and " $s$ " denote directions outward normal and tangent to the boundary, respectively. Each region with an area of $A^{(k)}$ can be under uniform temperature change, $T^{(k)}$. The thickness of the laminates (regions) is denoted by $h^{(k)}$. The contact region between the $\ell^{\text {th }}$ bolt and the hole boundary in the $k^{\text {th }}$ region is denoted by $\hat{\Gamma}^{(k \ell)}$. The sub-or superscripts " $(k)$ " and " $(\ell)$ " refer to the regions (laminates) and bolts, respectively. Their ranges are specified by $k=1, \ldots, K$ and $\ell=1, \ldots, L$, with $K$ and $L$ being the total number of regions (laminates) and bolts, respectively. As illustrated in Fig. 2, the hole radius, $a_{\ell}$, which is slightly larger than the bolt radius, $R_{\ell}$, leads to a clearance of $\delta_{\ell}$. The hole and bolt radii remain the same for each region. As shown in Fig. 2, the center of each hole, located at $\left(X_{\ell}, Y_{\ell}\right)$, coincides with the origin of the Cartesian coordinates $\left(x_{\ell}, y_{\ell}\right)$.
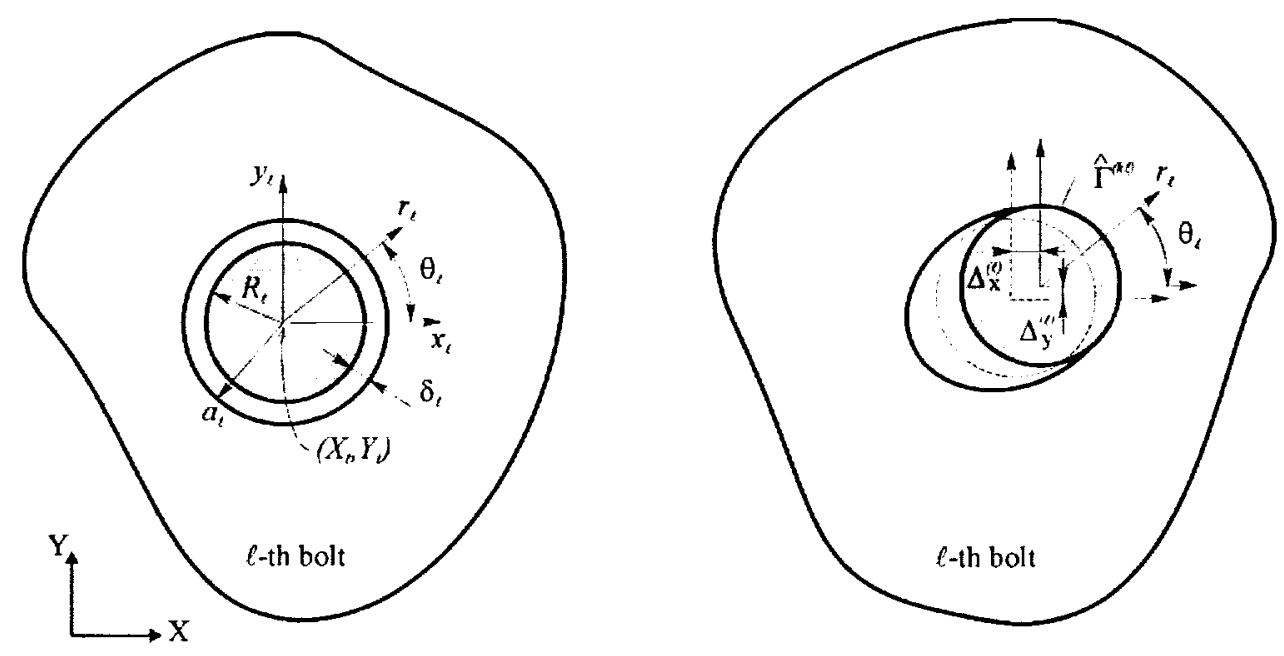

Fig. 2. Position of a bolt before and after the load is executed.

As shown in Fig. 3, the free-body diagram of each component of a lap joint, the unknown boundary traction components, $\tilde{\lambda}_{\rho}^{(k)}$, arise from the deformation of the boundary given by $\tilde{c}_{\rho}^{(k)}=\left(u_{\rho}^{(k)}-\tilde{u}_{\rho}^{(k)}\right)$ along a portion of the external boundary, $\tilde{\Gamma}^{(k)}$. The unknown traction component in the outward normal direction, $\hat{\lambda}_{n}^{(k \ell)}$, arises from the deformation of the contact zone between the $\ell^{\text {th }}$ bolt and the hole boundary in the $k^{\text {th }}$ region laminate. This contact zone deformation is expressed by $\hat{c}^{(k \ell)}=$ $u_{n}^{(k)}-\hat{u}_{n}^{(k \ell)}\left(\Delta_{j}^{(\ell)}\right)-\delta_{(k \ell)}^{*}$ along the contact region denoted by $\hat{\Gamma}^{(k \ell)}$. The extent of the contact region denoted by $\hat{\Gamma}^{(k \ell)}$ is dependent on the bolt displacement, $\hat{u}_{n}^{(k \ell)}\left(\Delta_{j}^{(\ell)}\right)$, deformation of the hole boundary, 


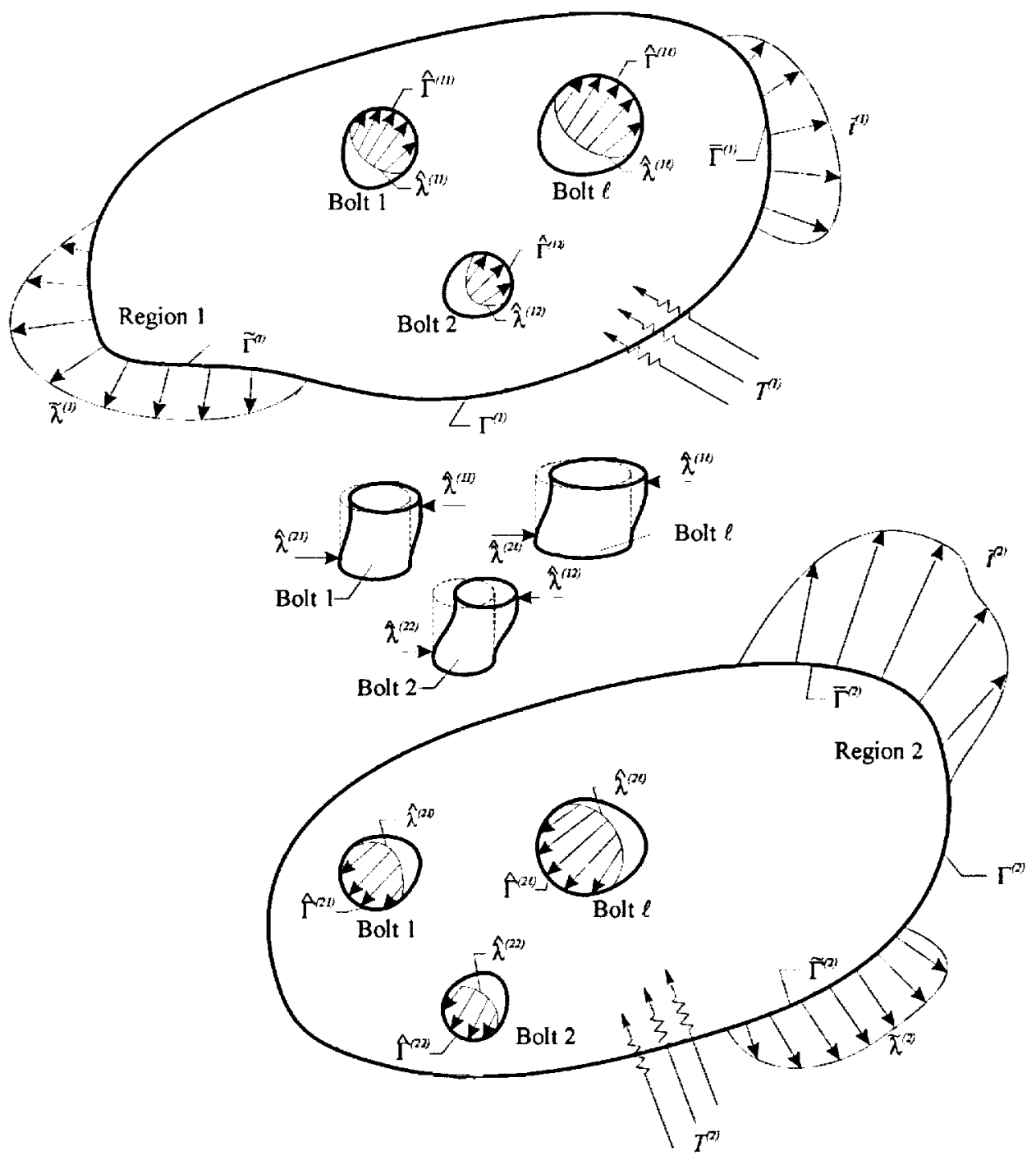

Fig. 3. Free-body diagram of each component in a bolted single-lap joint.

$u_{n}^{(k)}$, and the gap, $\delta_{(k \ell)}^{*}$. Because of the absence of friction between the bolt and the laminate, the tangential component of the bolt displacement, $\hat{u}_{s}^{(k \ell)}$, and the traction vector, $\hat{\lambda}_{s}^{(k \ell)}$, vanish, i.e. $\hat{u}_{s}^{(k \ell)}=0$ and $\hat{\lambda}_{s}^{(k \ell)}=0$. As shown in Fig. 4, at the point of initial contact (prior to any deformation of hole boundary), the gap between the hole boundary and the bolt (distance $\overline{P P^{\prime}}$ ) in the $k^{\text {th }}$ region is defined by

$$
\overline{P P^{\prime}}=\delta_{(k \ell)}^{*}=\delta_{\ell}\left[1-\cos \left(\theta-\theta^{*}\right)\right]
$$



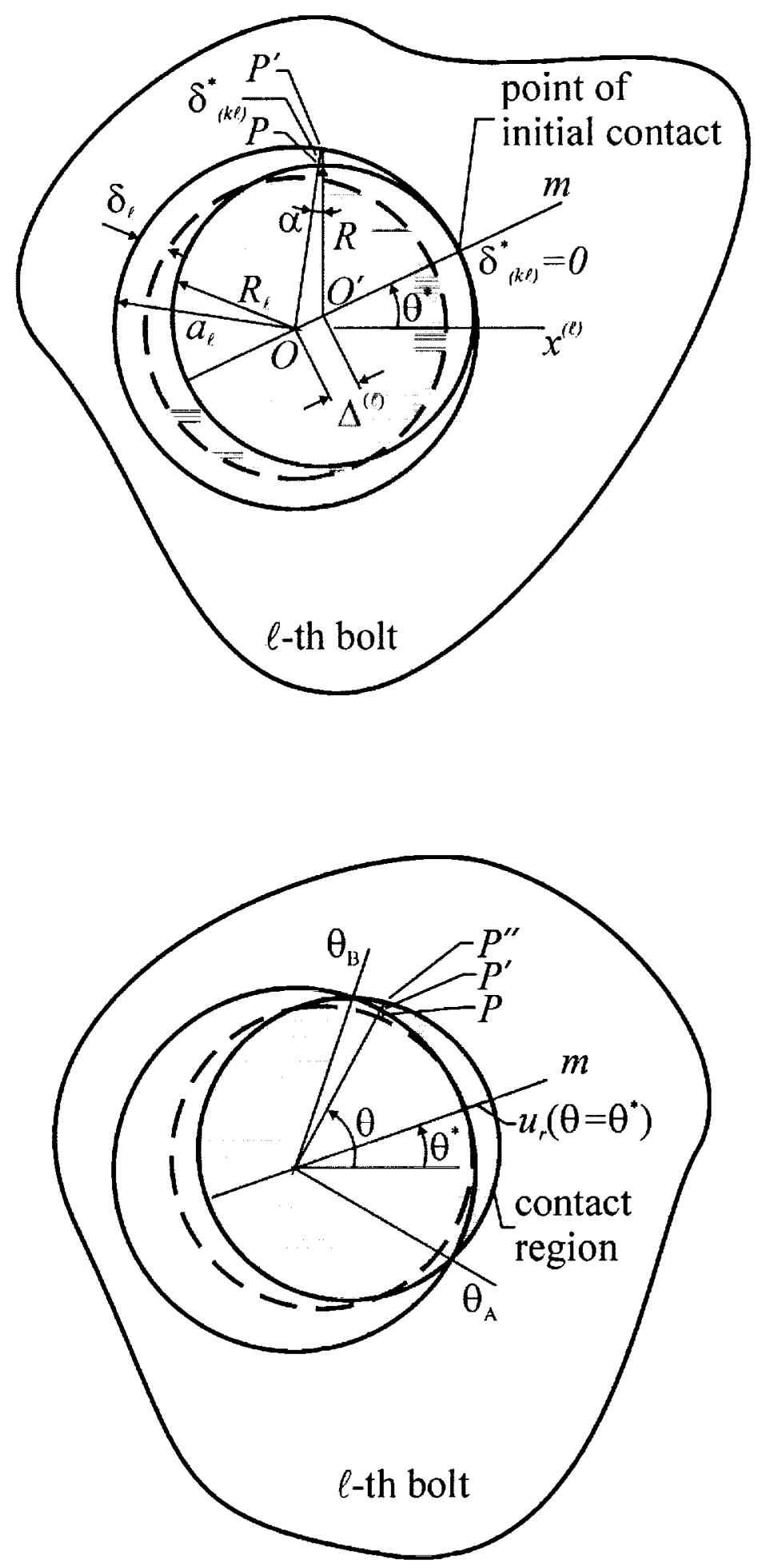

Fig. 4. The gap between the bolt and hole boundary immediately before and after the load is exerted. 
in which $\theta^{*}$ specifies the line of action, $m$, and $\delta_{\ell}$ is the clearance. The extent of the contact region, $\hat{\Gamma}^{(k \ell)}$, is defined by the angles $\theta_{A}$ and $\theta_{B}$. The flexible bolts experience deflections given by

$$
\begin{array}{ll}
\Delta^{(\ell)}=\left\{\Delta_{x 1}^{(\ell)}, \Delta_{x 2}^{(\ell)}, \Delta_{y 1}^{(\ell)}, \Delta_{y 2}^{(\ell)}\right\} & \text { for a single-lap joint } \\
\Delta^{(\ell)}=\left\{\Delta_{x 1}^{(\ell)}, \Delta_{x 2}^{(\ell)}, \Delta_{x 3}^{(\ell)}, \Delta_{y 1}^{(\ell)}, \Delta_{y 2}^{(\ell)}, \Delta_{y 3}^{(\ell)}\right\} & \text { for a double-lap joint }
\end{array}
$$

with $\Delta_{x i}^{(\ell)}$ and $\Delta_{y i}^{(\ell)}(i=1, K)$ representing the bolt deflection components at the $i^{\text {th }}$ point along the length of the $\ell^{\text {th }}$ bolt along the $x$ - and $y$-directions, respectively.

The material properties of each laminate are represented by the matrix $\mathbf{A}^{(k)}$ relating the stress resultants, $N_{\alpha \beta}^{(k)}$, to strain resultants, $\varepsilon_{\alpha \beta}^{(k)}$, with $\alpha, \beta=x, y$, in the form

$$
\left\{\begin{array}{l}
N_{x x}^{(k)} \\
N_{y y}^{(k)} \\
N_{x y}^{(k)}
\end{array}\right\}=\left[\begin{array}{lll}
A_{11} & A_{12} & A_{16} \\
A_{12} & A_{22} & A_{26} \\
A_{16} & A_{26} & A_{66}
\end{array}\right]^{(k)}\left\{\begin{array}{c}
\varepsilon_{x x}^{(k)} \\
\varepsilon_{y y}^{(k)} \\
\varepsilon_{x y}^{(k)}
\end{array}\right\} \quad \text { or } \mathbf{N}^{(k)}=\mathbf{A}^{(k)} \varepsilon^{(k)}
$$

where $A_{i j}^{(k)}$ are the components of the in-plane stiffness matrix $\mathbf{A}^{(k)}$ of the $k^{\text {th }}$ region. The strain components arising from temperature change, ${ }^{*} \varepsilon_{\alpha \beta}^{(k)}$, are expressed as

$$
\left({ }^{*} \varepsilon_{x x}^{(k)},{ }^{*} \varepsilon_{y y}^{(k)}, \gamma_{x y}^{(k)}\right)=\left(\alpha_{x x}^{(k)}, \alpha_{y y}^{(k)}, \alpha_{x y}^{(k)}\right) T^{(k)}
$$

where the coefficients $\alpha_{x x}^{(k)}, \alpha_{y y}^{(k)}$, and $\alpha_{x y}^{(k)}$ represent the thermal expansions of the $k^{\text {th }}$ region with respect to the global $(X, Y)$ coordinate system. The corresponding thermal stress resultants are defined as ${ }^{*} N_{\alpha \beta}^{(k)}$

$$
\left\{\begin{array}{c}
{ }^{*} N_{x x}^{(k)} \\
{ }^{*} N_{y y}^{(k)} \\
{ }^{*} N_{x y}^{(k)}
\end{array}\right\}=\left[\begin{array}{lll}
A_{11} & A_{12} & A_{16} \\
A_{12} & A_{22} & A_{26} \\
A_{16} & A_{26} & A_{66}
\end{array}\right]^{(k)}\left\{\begin{array}{c}
\alpha_{x x}^{(k)} \\
\alpha_{y y}^{(k)} \\
\alpha_{x y}^{(k)}
\end{array}\right\} T^{(k)}
$$

In matrix form, this relationship is expressed as

$$
{ }^{*} \mathbf{N}^{(k)}=\mathbf{A}^{(k) *} \boldsymbol{\varepsilon}^{(k)}
$$

in which ${ }^{*} \varepsilon^{(k)}=\boldsymbol{\alpha}^{(k)} T^{(k)}$, with $\boldsymbol{\alpha}^{(k)^{T}}=\left\{\alpha_{x x}^{(k)}, \alpha_{y y}^{(k)}, \alpha_{x y}^{(k)}\right\}$. 
The stiffness matrix of the bolts, $\mathbf{b}^{(\ell)}$, is given by

$$
\mathbf{b}^{(\ell)}=\left[\begin{array}{cc}
\mathbf{b}_{x}^{(\ell)} & 0 \\
0 & \mathbf{b}_{y}^{(\ell)}
\end{array}\right]
$$

whose coefficients are determined by modeling the bolt as a beam under concentrated forces. The explicit expressions for bolt stiffness for a single- and double-lap joint, as well as the general lap configurations, are derived in Appendix A.

These angles, the contact stresses, the components of bolt displacement, and the forces exerted by the bolts are the unknowns to be determined as part of the solution. Unless indicated otherwise, the subscripts $\alpha$ and $\beta$ vary as $\alpha, \beta=x, y$, representing the $(x, y)$ global coordinates. The subscript $\rho$ varies as $\rho=n, s$, representing the directions normal and tangent to the boundary, as shown in Fig. 1. Also, only repeated subscripts imply summation.

\section{SOLUTION METHOD}

The solution method is based on the variational formulation in conjunction with the complex potential theory. The governing equations are derived by requiring the first variation of the total potential energy, arising from thermal and mechanical loads, to vanish. The in-plane equilibrium equations in each region are satisfied exactly by employing complex potential functions in the form suggested by Lekhnitskii (1968). However, each of the bolt equilibrium equations and the boundary conditions are satisfied by minimizing the total potential energy.

\subsection{Governing Equations}

The total potential energy for $K$ regions connected with $L$ number of bolts in the absence of friction between the laminates and between the laminates and the bolts along the contact region under mechanical and thermal loading can be expressed as

$$
\pi=\sum_{k=1}^{K} U^{(k)}+\sum_{\ell=1}^{L} B^{(\ell)}+\sum_{k=1}^{K} \sum_{\ell=1}^{L} \hat{W}^{(k \ell)}+\sum_{k=1}^{K} \tilde{W}^{(k)}+\sum_{k=1}^{K} \bar{W}^{(k)}
$$

The strain energy of the $k^{\text {th }}$ laminate, $U^{(k)}$, is given by

$$
U^{(k)}=\frac{1}{2} \int_{A^{(k)}} N_{\alpha \beta}^{(k)} \varepsilon_{\alpha \beta}^{(k)} d A-\int_{A^{(k)}} N_{\alpha \beta}^{(k) *} \varepsilon_{\alpha \beta}^{(k)} d A
$$


with its first variation as derived in Appendix B,

$$
\begin{aligned}
& \delta U^{(k)}=-\int_{A^{(k)}} N_{\alpha \beta, \beta}^{(k)} \delta u_{\alpha}^{(k)} d A+\int_{\bar{\Gamma}^{(k)}} t_{\alpha}^{(k)} \delta u_{\alpha}^{(k)} d \Gamma \\
& +\int_{\tilde{\Gamma}^{(k)}}^{*} t_{\rho}^{(k)} \delta u_{\rho}^{(k)} d \Gamma+\sum_{\ell=1}^{L^{(k)}} \int_{\Gamma^{(k \ell)}}^{*} t_{\rho}^{(k)} \delta u_{\rho}^{(k)} d \Gamma
\end{aligned}
$$

in which $\alpha, \beta=x, y$ and $\rho=n, s$. The strain energy of the $\ell^{\text {th }}$ bolt, $B^{(\ell)}$, is given by

$$
B^{(\ell)}=\frac{1}{2} \Delta_{i}^{(\ell)} b_{i j}^{(\ell)} \Delta_{j}^{(\ell)} \text { with } i, j=1,2 K
$$

with its first variation

$$
\delta B^{(\ell)}=b_{i j}^{(\ell)} \Delta_{j}^{(\ell)} \delta \Delta_{i}^{(\ell)}
$$

The potential of the reaction forces, $\hat{\lambda}_{n}^{(k \ell)}$ and $\tilde{\lambda}_{\rho}^{(k)}(\rho=n, s)$, arising from the contact between the bolt and the hole boundary and the applied displacement constraints along the external boundary are denoted by $\hat{W}^{(k \ell)}$ and $\tilde{W}^{(k)}$, respectively. They are expressed in the form

in which

$$
\hat{W}^{(k \ell)}=\int_{\hat{\Gamma}^{(k \ell)}} \hat{J}^{(k \ell)} \hat{\lambda}_{n}^{(k \ell)}\left\{u_{n}^{(k)}-\hat{u}_{n}^{(k \ell)}\left(\Delta_{j}^{(\ell)}\right)-\delta_{(k \ell)}^{*}\right\} d \Gamma
$$

$$
\hat{J}^{(k \ell)}= \begin{cases}1 & \text { contact between the } k^{\text {th }} \text { plate and } \ell^{\text {th }} \text { bolt } \\ 0 & \text { no contact between the } k^{\text {th }} \text { plate and } \ell^{\text {th }} \text { bolt }\end{cases}
$$

and

$$
\tilde{W}^{(k)}=\int_{\bar{\Gamma}^{(k)}} \tilde{\lambda}_{\rho}^{(k)}\left\{u_{\rho}^{(k)}-\tilde{u}_{\rho}^{(k)}\right\} d \Gamma \text { with } \rho=n, s
$$

The potential of the externally applied tractions, $\bar{t}_{\alpha}^{(k)}$, denoted by $\bar{W}^{(k)}$ is expressed as

$$
\bar{W}^{(k)}=-\int_{\bar{\Gamma}^{(k)}} \bar{t}_{\alpha}^{(k)} u_{\alpha}^{(k)} d \Gamma \text { with } \alpha=x, y
$$

in which $\bar{t}_{\alpha}^{(k)}$ and $u_{\alpha}^{(k)}$ represent the applied traction and displacement components in the $x$ - and $y$ directions, respectively. Their first variations are obtained as 


$$
\begin{aligned}
& \delta \hat{W}^{(k \ell)}=\int_{\hat{\Gamma}^{(k \ell)}} \hat{J}^{(k \ell)}\left\{u_{n}^{(k)}-\hat{u}_{n}^{(k \ell)}\left(\Delta_{j}^{(\ell)}\right)-\delta_{(k \ell)}^{*}\right\} \delta \hat{\lambda}_{n}^{(k \ell)} d \Gamma \\
& \quad+\int_{\hat{\Gamma}^{(k \ell)}} \hat{J}^{(k \ell)} \hat{\lambda}_{n}^{(k \ell)} \delta u_{n}^{(k)} d \Gamma-\int_{\hat{\Gamma}^{(k \ell)}} \hat{J}^{(k \ell)} \hat{\lambda}_{n}^{(k \ell)} \frac{\partial \hat{u}_{n}^{(k \ell)}}{\partial \Delta_{i}^{(\ell)}} \delta \Delta_{i}^{(\ell)} d \Gamma
\end{aligned}
$$

and

$$
\delta \tilde{W}^{(k)}=\int_{\tilde{\Gamma}^{(k)}} \delta \tilde{\lambda}_{\rho}^{(k)}\left\{u_{\rho}^{(k)}-\tilde{u}_{\rho}^{(k)}\right\} d \Gamma+\int_{\tilde{\Gamma}^{(k)}} \tilde{\lambda}_{\rho}^{(k)} \delta u_{\rho}^{(k)} d \Gamma
$$

and

$$
\delta \bar{W}^{(k)}=-\int_{\bar{\Gamma}^{(k)}} \bar{t}_{\alpha}^{(k)} \delta u_{\alpha}^{(k)} d \Gamma
$$

The first variation of the total potential energy can be obtained as

$$
\begin{aligned}
\delta \pi & =-\sum_{k=1}^{K} \int_{A^{(k)}} N_{\alpha \beta, \beta}^{(k)} \delta u_{\alpha}^{(k)} d A+\sum_{\ell=1}^{L}\left\{b_{i j}^{(\ell)} \Delta_{j}^{(\ell)}-\sum_{k=1}^{K} \int_{\hat{\Gamma}^{(k \ell)}} \hat{J}^{(k \ell)} \hat{\lambda}_{n}^{(k \ell)} \frac{\partial \hat{u}_{n}^{(k \ell)}}{\partial \Delta_{i}^{(\ell)}} d \Gamma\right\} \delta \Delta_{i}^{(\ell)} \\
& +\sum_{k=1}^{K} \int_{\bar{\Gamma}^{(k)}}\left\{t_{\alpha}^{(k)}-\bar{t}_{\alpha}^{(k)}\right\} \delta u_{\alpha}^{(k)} d \Gamma+\sum_{k=1}^{K} \sum_{\ell=1}^{L} \int_{\hat{\Gamma}^{(k \ell)}}\left({ }^{*} t_{n}^{(k)}+\hat{J}^{(k \ell)} \hat{\lambda}_{n}^{(k \ell)}\right) \delta u_{n}^{(k)} d \Gamma \\
& +\sum_{k=1}^{K} \sum_{\ell=1}^{L} \int_{\hat{\Gamma}^{(k \ell)}}{ }^{*} t_{s}^{(k)} \delta u_{s}^{(k)} d \Gamma+\sum_{k=1}^{K} \int_{\tilde{\Gamma}^{(k)}}\left\{{ }^{*} t_{\rho}^{(k)}+\tilde{\lambda}_{\rho}^{(k)}\right\} \delta u_{\rho}^{(k)} d \Gamma+\sum_{k=1}^{K} \int_{\tilde{\Gamma}^{(k)}}\left\{u_{\rho}^{(k)}-\tilde{u}_{\rho}^{(k)}\right\} \delta \tilde{\lambda}_{\rho}^{(k)} d \Gamma \\
& +\sum_{k=1}^{K} \sum_{\ell=1}^{L} \int_{\hat{\Gamma}^{(k \ell)}} \hat{J}^{(k \ell)}\left\{u_{n}^{(k)}-\hat{u}_{n}^{(k \ell)}\left(\Delta_{j}^{(\ell)}\right)+\delta_{(k \ell)}^{*}\right\} \delta \hat{\lambda}_{n}^{(k \ell)} d \Gamma
\end{aligned}
$$

Noting that $\delta u_{\alpha}^{(k)}, \delta u_{\rho}^{(k)}, \delta \hat{u}_{n}^{(k)}, \delta \hat{u}_{s}^{(k)}, \delta \Delta_{i}^{(\ell)}$, and $\delta \hat{\lambda}_{n}^{(k \ell)}$ are arbitrary independent quantities and requiring the first variation of the total potential energy to vanish lead to the equilibrium equations for the laminates and bolts as

$$
\begin{gathered}
N_{\alpha \beta, \beta}^{(k)}=0 \quad \text { on } A^{(k)} \\
b_{i j}^{(\ell)} \Delta_{j}^{(\ell)}-\sum_{k=1}^{K} \int_{\hat{\Gamma}^{(k \ell)}} \hat{J}^{(k \ell)} \hat{\lambda}_{n}^{(k \ell)} \frac{\partial \hat{u}_{n}^{(k \ell)}}{\partial \Delta_{i}^{(\ell)}} d \Gamma=0 \quad i, j=1,2 K
\end{gathered}
$$


Their associated boundary conditions are obtained as

$$
\begin{gathered}
\left\{{ }^{*} t_{\alpha}^{(k)}-\bar{t}_{\alpha}^{(k)}\right\}=0 \text { on } \bar{\Gamma}^{(k)} \\
\left({ }^{*} t_{n}^{(k)}+\hat{J}^{(k \ell)} \hat{\lambda}_{n}^{(k \ell)}\right)=0 \text { on } \hat{\Gamma}^{(k \ell)} \\
{ }^{*}{ }_{s}^{(k)}=0 \text { on } \hat{\Gamma}^{(k \ell)} \\
\left\{{ }^{*} t_{\rho}^{(k)}+\tilde{\lambda}_{\rho}^{(k)}\right\}=0 \text { on } \tilde{\Gamma}^{(k)} \\
\left\{u_{\rho}^{(k)}-\tilde{u}_{\rho}^{(k)}\right\}=0 \text { on } \tilde{\Gamma}^{(k)} \\
\hat{J}^{(k \ell)}\left\{u_{n}^{(k)}-\hat{u}_{n}^{(k \ell)}\left(\Delta_{j}^{(\ell)}\right)+\delta_{(k \ell)}^{*}\right\}=0 \text { on } \hat{\Gamma}^{(k \ell)}
\end{gathered}
$$

where $\alpha, \beta=x, y ; \rho=n, s ; k=1, K$; and $\ell=1, \ldots, L$.

\subsection{Total Potential Energy}

The strain energy expression given in Eq. (9) can be rewritten in terms of displacement components as

$$
U^{(k)}=\frac{1}{2} \int_{A^{(k)}} N_{\alpha \beta}^{(k)} u_{\alpha, \beta}^{(k)} d A^{(k)}-\int_{A^{(k)}}^{*} N_{\alpha \beta}^{(k)} u_{\alpha, \beta}^{(k)} d A
$$

Its integration by parts yields

$$
U^{(k)}=-\frac{1}{2}\left(N_{\alpha \beta, \beta}^{(k)}-2^{*} N_{\alpha \beta, \beta}^{(k)}\right) u_{\alpha}^{(k)} d A+\frac{1}{2} \int_{A^{(k)}}\left[\left(N_{\alpha \beta}^{(k)}-2^{*} N_{\alpha \beta}^{(k)}\right) u_{\alpha}^{(k)}\right]_{, \beta} d A
$$

Under uniform temperature distribution and applying Gauss' theorem, it reduces to

$$
U^{(k)}=-\frac{1}{2} \int_{A^{(k)}} N_{\alpha \beta, \beta}^{(k)} u_{\alpha}^{(k)} d A+\frac{1}{2} \int_{\Gamma^{(k)}}\left(N_{\alpha \beta}^{(k)}-2^{*} N_{\alpha \beta}^{(k)}\right) n_{\beta}^{(k)} u_{\alpha}^{(k)} d \Gamma
$$

This expression can be further simplified by invoking the stress resultants, $N_{\alpha \beta, \beta}$, that satisfy the equilibrium equations, $N_{\alpha \beta, \beta}=0$, given by Eq. (20), as

$$
U^{(k)}=\frac{1}{2} \int_{\Gamma^{(k)}}\left(N_{\alpha \beta}^{(k)}-2^{*} N_{\alpha \beta}^{(k)}\right) n_{\beta}^{(k)} u_{\alpha}^{(k)} d \Gamma
$$


The explicit expressions for the stress resultants and displacement components satisfying both the equilibrium equations and the compatibility conditions are given in Appendix C.

Finally, the total potential energy expression given in Eq. (19) is reduced to a form, free of area integrals, as

$$
\begin{aligned}
\pi & =\sum_{k=1}^{K} \frac{1}{2} \int_{\Gamma^{(k)}}\left(N_{\alpha \beta}^{(k)}-2^{*} N_{\alpha \beta}^{(k)}\right) n_{\beta}^{(k)} u_{\alpha}^{(k)} d \Gamma+\frac{1}{2} \sum_{\ell=1}^{L} b_{i j}^{(\ell)} \Delta_{i}^{(\ell)} \Delta_{j}^{(\ell)}+\sum_{k=1}^{N} \int_{\tilde{\Gamma}^{(k)}} \tilde{\lambda}_{\rho}^{(k)}\left\{u_{\rho}^{(k)}-\tilde{u}_{\rho}^{(k)}\right\} d \Gamma \\
& +\sum_{k=1}^{K} \sum_{\ell=1}^{L} \int_{\hat{\Gamma}^{(k \ell)}} J^{(k \ell)} \hat{\lambda}_{n}^{(k \ell)}\left\{u_{n}^{(k)}-\hat{u}_{n}^{(k \ell)}\left(\Delta_{j}^{(\ell)}\right)+\delta_{(k \ell)}^{*}\right\} d \Gamma-\sum_{k=1}^{K} \int_{\Gamma^{(k)}} \bar{t}_{\alpha}^{(k)} u_{\alpha}^{(k)} d \Gamma
\end{aligned}
$$

with $\alpha, \beta=x, y ; \rho=n, s ; k=1, \ldots, K$; and $\ell=1, \ldots, L$. It can be rewritten in matrix form as

$$
\begin{aligned}
\pi & =\sum_{k=1}^{K} \frac{1}{2} \int_{\Gamma^{(k)}}\left(\mathbf{N}^{(k)}-2^{*} \mathbf{N}^{(k)}\right)^{T} \mathbf{n}^{(k)} \mathbf{u}^{(k)} d \Gamma+\frac{1}{2} \sum_{\ell=1}^{L} \mathbf{b}^{(\ell)} \Delta^{(\ell)}+\sum_{k=1}^{K} \int_{\Gamma^{(k)}} \tilde{\lambda}^{(k)^{T}} \tilde{\mathbf{c}}^{(k)} d \Gamma \\
& +\sum_{k=1}^{K} \sum_{\ell=1}^{L} \int_{\hat{\Gamma}^{(k \ell)}} \hat{J}^{(k \ell)} \hat{\lambda}^{(k \ell)^{T}} \hat{\mathbf{c}}^{(k \ell)} d \Gamma-\sum_{k=1}^{K} \int_{\Gamma^{(k)}} \overline{\mathbf{t}}^{(k)^{T}} \mathbf{u}^{(k)} d \Gamma
\end{aligned}
$$

where

$$
\begin{aligned}
& \tilde{\mathbf{c}}^{(k)}=\mathbf{u}^{(k)}-\tilde{\mathbf{u}}^{(k)} ; \hat{\mathbf{c}}^{(k \ell)}=\mathbf{u}^{(k)}-\hat{\mathbf{u}}^{(k \ell)}\left(\Delta^{(\ell)}\right)-\boldsymbol{\delta}_{(k \ell)}^{*} \\
& \mathbf{u}^{(k)^{T}}=\left\{u_{x}^{(k)}, u_{y}^{(k)}\right\} ; \tilde{\mathbf{u}}^{(k)^{T}}=\left\{\tilde{u}_{n}^{(k)}, \tilde{u}_{s}^{(k)}\right\} ; \hat{\mathbf{u}}^{(k)^{T}}=\left\{\hat{u}_{n}^{(k)}, 0\right\} \\
& \overline{\mathbf{t}}^{(k)^{T}}=\left\{\bar{t}_{x}^{(k)}, \bar{t}_{y}^{(k)}\right\} ; \quad \tilde{\lambda}^{(k)^{T}}=\left\{\tilde{\lambda}_{n}^{(k)}, \tilde{\lambda}_{s}^{(k)}\right\} ; \hat{\lambda}^{(k \ell)}=\left\{\hat{\lambda}_{n}^{(k \ell)}, 0\right\} \\
& \tilde{\mathbf{c}}^{(k)^{T}}=\left\{\tilde{c}_{n}^{(k)}, \tilde{c}_{s}^{(k)}\right\} ; \hat{\mathbf{c}}^{(k \ell)^{T}}=\left\{\hat{c}_{n}^{(k \ell)}, 0\right\} ; \quad \boldsymbol{\delta}_{(k \ell)}^{*}{ }^{T}=\left\{\delta_{(k \ell)}^{*}, 0\right\}
\end{aligned}
$$

and the matrix of unit normals, $\mathbf{n}^{(k)}$, is expressed as

$$
\mathbf{n}^{(k)}=\left[\begin{array}{cc}
n_{x}^{(k)} & 0 \\
0 & n_{y}^{(k)} \\
n_{y}^{(k)} & n_{x}^{(k)}
\end{array}\right]
$$

As given by Equation (3), the unknown bolt displacements are contained in vector $\Delta^{(\ell)}$, and matrix $\mathbf{b}^{(\ell)}$ represents the bolt stiffness. 
The constraint condition given by Equation (22e),

$$
\tilde{c}_{\rho}^{(k)}=u_{\rho}^{(k)}-\tilde{u}_{\rho}^{(k)}=0 \text { with } \rho=n, s
$$

is rewritten in vector form as

$$
\tilde{\mathbf{c}}^{(k)}=\mathbf{u}^{\prime(k)}-\tilde{\mathbf{u}}^{\prime(k)}=\mathbf{0}
$$

in which

$$
\mathbf{u}^{\prime(k)}=\mathbf{T}^{(k)} \mathbf{u}^{(k)}
$$

with $\mathbf{T}^{(k)}$ representing the transformation matrix between the $(x, y)$ and $(n, s)$ coordinate systems. The displacement vectors $\mathbf{u}^{\prime(k)}$ and $\mathbf{u}^{(k)}$ are defined in the $(n, s)$ and $(x, y)$ coordinate systems, respectively,

$$
\begin{aligned}
& \mathbf{u}^{(k)^{T}}=\left\{u_{n}^{(k)}, u_{s}^{(k)}\right\} \\
& \mathbf{u}^{(k)^{T}}=\left\{u_{x}^{(k)}, u_{y}^{(k)}\right\}
\end{aligned}
$$

Substituting for $\mathbf{u}^{(k)}$ from Equation (B31) into Equation (32) yields

$$
\mathbf{u}^{(k)}=\mathbf{T}^{(k)} \mathbf{U}^{(k)^{T}} \mathbf{a}^{(k)}
$$

leading to

$$
\tilde{\mathbf{c}}^{(k)}=\mathbf{T}^{(k)} \mathbf{U}^{(k)^{T}} \mathbf{a}^{(k)}-\tilde{\mathbf{u}}^{(k)}
$$

The unknown traction vector, $\tilde{\lambda}^{(k)}$, defined in Eq. (29c) can be assumed as

$$
\tilde{\lambda}^{(k)}=\sum_{j=0}^{J} \mathbf{P}_{j} \tilde{\mathbf{\Lambda}}_{j}^{(k)}
$$

where the matrix $\mathbf{P}_{j}$ and vector of unknown coefficients $\tilde{\Lambda}_{j}^{(k)}$ are defined as

$$
\mathbf{P}_{j}=\left[\begin{array}{cc}
p_{j} & 0 \\
0 & p_{j}
\end{array}\right] \text { and } \tilde{\Lambda}_{j}^{(k)^{T}}=\left\{\tilde{\Lambda}_{n j}, \tilde{\Lambda}_{s j}\right\}
$$

with $p_{j}$ being the $j^{\text {th }}$-order Legendre polynomial. In matrix notation, this equation becomes 
where

$$
\tilde{\lambda}^{(k)}=\mathbf{P} \tilde{\Lambda}^{(k)}
$$

$$
\mathbf{P}=\left[\begin{array}{lllll}
\mathbf{P}_{0} & \mathbf{P}_{1} & \mathbf{P}_{2} & \cdots & \mathbf{P}_{J}
\end{array}\right] \text { and } \tilde{\Lambda}^{(k)^{T}}=\left\{\tilde{\Lambda}_{0}^{T}, \tilde{\Lambda}_{1}^{T}, \tilde{\Lambda}_{2}^{T}, \ldots, \tilde{\Lambda}_{J}^{T}\right\}
$$

From Eqs. (36) and (40), the boundary integral of the product $\tilde{\lambda}^{(k)^{T}} \tilde{\mathbf{c}}^{(k)}$ that appears in the expression for the total potential energy is obtained as

$$
\begin{aligned}
\int_{\tilde{\Gamma}^{(k)}} \tilde{\lambda}^{(k)^{T}} \tilde{\mathbf{c}}^{(k)} d \Gamma & =\int_{\tilde{\Gamma}^{(k)}} \tilde{\mathbf{\Lambda}}^{(k)^{T}} \mathbf{P}^{T} \mathbf{T}^{(k)} \mathbf{U}^{(k)} \mathbf{a}^{(k)} d \Gamma-\int_{\tilde{\Gamma}^{(k)}} \tilde{\mathbf{\Lambda}}^{(k)^{T}} \mathbf{P}^{T} \mathbf{T}^{(k)} \tilde{\mathbf{u}}^{(k)} d \Gamma \\
& =\tilde{\boldsymbol{\Lambda}}^{(k)^{T}} \tilde{\mathbf{C}}^{(k)} \mathbf{a}^{(k)}-\tilde{\boldsymbol{\Lambda}}^{(k)^{T}} \tilde{\mathbf{f}}^{(k)}
\end{aligned}
$$

where

$$
\tilde{\mathbf{C}}^{(k)}=\int_{\tilde{\Gamma}^{(k)}} \mathbf{P}^{T} \mathbf{T}^{(k)} \mathbf{U}^{(k)^{T}} d \Gamma
$$

and

$$
\tilde{\mathbf{f}}^{(k)}=\int_{\tilde{\Gamma}^{(k)}} \mathbf{P}^{T} \mathbf{T}^{(k)} \tilde{\mathbf{u}}^{(k)} d \Gamma
$$

The constraint condition given by Equation (22f),

$$
\hat{c}^{(k \ell)}=u_{n}^{(k)}-\hat{u}_{n}^{(k \ell)}\left(\Delta_{j}^{(\ell)}\right)+\delta_{(k \ell)}^{*}=0
$$

is rewritten in vector form as

$$
\hat{\mathbf{c}}^{(k \ell)}=\mathbf{u}^{\prime(k)}-\hat{\mathbf{u}}^{(k \ell)}\left(\Delta^{(\ell)}\right)+\boldsymbol{\delta}_{(k \ell)}^{*}=0
$$

The bolt displacement vector, $\hat{\mathbf{u}}^{(k \ell)}$, at the contact region can be expressed in terms of the bolt deflections in the form

$$
\hat{\mathbf{u}}^{\prime(k \ell)}=\hat{\mathbf{G}}^{(k \ell)} \Delta^{(\ell)}
$$

in which the matrix $\hat{\mathbf{G}}^{(k \ell)}$ and the vector $\Delta^{(\ell)}$ are defined as 


$$
\hat{\mathbf{G}}^{(k \ell)}=\left[\begin{array}{cc}
\sin \theta_{k l} & \cos \theta_{k l} \\
0 & 0
\end{array}\right]\left[\begin{array}{ccccc}
\eta\left(z_{1}-z_{k}\right) & \eta\left(z_{2}-z_{k}\right) & \cdots & \eta\left(z_{K}-z_{k}\right) & \\
0 & 0 & \cdots & 0 & \\
& 0 & 0 & \cdots & 0 \\
& \eta\left(z_{1}-z_{k}\right) & \eta\left(z_{2}-z_{k}\right) & \cdots & \eta\left(z_{K}-z_{k}\right)
\end{array}\right]
$$

with $\eta\left(z_{i}-z_{k}\right)$ defined as

$$
\eta\left(z_{i}-z_{k}\right)=\left\{\begin{array}{l}
1 \text { if } i=k \\
0 \text { if } i \neq k
\end{array} \quad(i=1, \ldots, K)\right.
$$

and

$$
\Delta^{(\ell)^{T}}=\left\{\begin{array}{llllllll}
\Delta_{x 1}^{(\ell)} & \Delta_{x 2}^{(\ell)} & \cdots & \Delta_{x K}^{(\ell)} & \Delta_{y 1}^{(\ell)} & \Delta_{y 2}^{(\ell)} & \cdots & \Delta_{y K}^{(\ell)}
\end{array}\right\}
$$

With substitutions from Eq. (35) and (46), the expression for $\hat{\mathbf{c}}^{(k \ell)}$ becomes

$$
\hat{\mathbf{c}}^{(k \ell)}=\mathbf{T}^{(k)} \mathbf{U}^{(k)}{ }^{T} \mathbf{a}^{(k)}-\hat{\mathbf{G}}^{(k \ell)} \Delta^{(\ell)}+\boldsymbol{\delta}_{(k \ell)}^{*}
$$

The unknown traction vector, $\hat{\lambda}^{(k \ell)}$, defined in Eq. (29c) can be assumed as

$$
\hat{\lambda}^{(k \ell)}=\sum_{i=0}^{I} \mathbf{F}_{i} \hat{\Lambda}_{i}^{(k \ell)}
$$

where the matrix $\mathbf{F}_{i}$ and vector of unknown coefficients $\hat{\Lambda}_{i}^{(k \ell)}$ are defined as

$$
\mathbf{F}_{i}=\left[\begin{array}{cc}
F_{i} & 0 \\
0 & F_{i}
\end{array}\right] \text { and } \hat{\Lambda}_{i}^{(k \ell)^{T}}=\left\{\hat{\Lambda}_{n i}^{(k \ell)}, 0\right\}
$$

in which $F_{i}$ is an orthogonal trigonometric function satisfying the condition of zero stress at the endpoints, i.e.,

$$
F_{i}\left(s=s_{0}\right)=F_{i}\left(s=s_{1}\right)=0
$$

These functions are formulated as

$$
F_{i}(s)=\sin \left[\frac{i \pi\left(\theta(s)-\theta\left(s_{0}\right)\right)}{\left(\theta\left(s_{1}\right)-\theta\left(s_{0}\right)\right)}\right] \text { with } i=1, I
$$

in which $\theta\left(s_{0}\right)$ and $\theta\left(s_{1}\right)$ represent the beginning and end angles of the contact region. These angles are measured with respect to the global $X$-axis, and any point in the contact region is identified by the angle $\theta(s)$. 
The expression for the unknown traction vector at the contact region can be rewritten as

$$
\hat{\boldsymbol{\lambda}}^{(k \ell)}=\mathbf{F} \hat{\boldsymbol{\Lambda}}^{(k \ell)}
$$

where

$$
\mathbf{F}=\left[\begin{array}{lllll}
\mathbf{F}_{0} & \mathbf{F}_{1} & \mathbf{F}_{2} & \cdots & \mathbf{F}_{l}
\end{array}\right] \text { and } \hat{\Lambda}^{(k \ell)^{T}}=\left\{\hat{\Lambda}_{0}^{(k \ell)}, \hat{\Lambda}_{1}^{(k \ell)}, \ldots, \hat{\Lambda}_{l}^{(k \ell)}\right\}
$$

From Eqs. (49) and (54), the boundary integral of the product $\hat{\lambda}^{(k)^{T}} \hat{\mathbf{c}}^{(k)}$ that appears in the expression for the total potential energy is obtained as

$$
\begin{aligned}
\int_{\hat{\Gamma}^{(k \ell)}} \hat{\lambda}^{(k \ell)^{T}} \hat{\mathbf{c}}^{(k \ell)} d \hat{\Gamma} & =\int_{\hat{\Gamma}^{(k \ell)}} \hat{\boldsymbol{\Lambda}}^{(k \ell)^{T}} \mathbf{F}^{T} \mathbf{T}^{(k)} \mathbf{U}^{(k)^{T}} \mathbf{a}^{(k)} d \Gamma-\int_{\hat{\Gamma}^{(k \ell)}} \hat{\boldsymbol{\Lambda}}^{(k \ell)^{T}} \mathbf{F}^{T} \hat{\mathbf{G}}^{(k \ell)} \Delta^{(\ell)} d \Gamma \\
& +\int_{\hat{\Gamma}^{(k \ell)}} \hat{\boldsymbol{\Lambda}}^{(k \ell)^{T}} \mathbf{F}^{T} \boldsymbol{\delta}_{(k \ell)}^{*} d \Gamma
\end{aligned}
$$

or

$$
\int_{\hat{\Gamma}^{(k \ell)}} \hat{\boldsymbol{\lambda}}^{(k \ell)^{T}} \hat{\mathbf{c}}^{(k \ell)} d \hat{\Gamma}=\hat{\boldsymbol{\Lambda}}^{(k \ell)} \hat{\mathbf{C}}^{(k \ell)} \mathbf{a}^{(k)}-\hat{\boldsymbol{\Lambda}}^{(k \ell)^{T}} \hat{\mathbf{g}}_{1}^{(k \ell)} \Delta^{(\ell)}+\hat{\boldsymbol{\Lambda}}^{(k \ell)^{T}} \hat{\mathbf{g}}_{0}^{(k \ell)}
$$

where

$$
\begin{gathered}
\hat{\mathbf{C}}^{(k \ell)}=\int_{\hat{\Gamma}^{(k \ell)}} \mathbf{F}^{T} \mathbf{T}^{(k)} \mathbf{U}^{(k)^{T}} d \Gamma \\
\hat{\mathbf{g}}_{1}^{(k \ell)}=\int_{\hat{\Gamma}^{(k \ell)}} \mathbf{F}^{T} \hat{\mathbf{G}}^{(k \ell)} d \Gamma \\
\hat{\mathbf{g}}_{0}^{(k \ell)}=\int_{\hat{\Gamma}^{(k \ell)}} \mathbf{F}^{T} \boldsymbol{\delta}_{(k \ell)}^{*} d \Gamma
\end{gathered}
$$

Also, the potential energy of the traction vector, $\overline{\mathbf{t}}^{(k)}$, acting on $\bar{\Gamma}^{(k)}$ is expressed as

$$
\int_{\bar{\Gamma}^{(k)}} \overline{\mathbf{t}}^{(k)^{T}} \mathbf{u}^{(k)} d \Gamma=\int_{\bar{\Gamma}^{(k)}} \mathbf{u}^{(k)^{T}} \overline{\mathbf{t}}^{(k)} d \Gamma=\int_{\bar{\Gamma}^{(k)}} \mathbf{a}^{(k)^{T}} \mathbf{U}^{(k)} \overline{\mathbf{t}}^{(k)} d \Gamma=\mathbf{a}^{(k)^{T}} \overline{\mathbf{f}}
$$

where 


$$
\overline{\mathbf{f}}=\int_{\bar{\Gamma}^{(k)}} \mathbf{U}^{(k)} \overline{\mathbf{t}}^{(k)} d \Gamma
$$

Substituting from Eqs. (B31), (41), (57), and (61) for the appropriate terms in Eq. (28), the expression for the total potential energy is obtained in matrix form as

$$
\begin{aligned}
\pi & =\frac{1}{2} \sum_{k=1}^{K} \mathbf{a}^{(k)^{T}} \mathbf{H}^{(k)} \mathbf{a}^{(k)}-\sum_{k=1}^{K}{ }^{*} \mathbf{h}^{(k)} \mathbf{a}^{(k)}+\frac{1}{2} \sum_{\ell=1}^{L} \Delta^{(\ell)^{T}} \mathbf{b}^{(\ell)} \Delta^{(\ell)} \\
& +\sum_{k=1}^{K} \tilde{\mathbf{\Lambda}}^{(k)^{T}} \tilde{\mathbf{C}}^{(k)} \mathbf{a}^{(k)}-\sum_{k=1}^{K} \tilde{\mathbf{\Lambda}}^{(k)^{T}} \tilde{\mathbf{f}}^{(k)} \\
& +\sum_{k=1}^{K} \sum_{\ell=1}^{L} \hat{\mathbf{\Lambda}}^{(k \ell)} \hat{\mathbf{C}}^{(k \ell)} \mathbf{a}^{(k)}-\sum_{k=1}^{K} \sum_{\ell=1}^{L} \hat{\mathbf{\Lambda}}^{(k \ell)^{T}} \hat{\mathbf{g}}_{1}^{(k \ell)} \mathbf{\Delta}^{(\ell)}+\sum_{k=1}^{K} \sum_{\ell=1}^{L} \hat{\mathbf{\Lambda}}^{(k \ell)^{T}} \hat{\mathbf{g}}_{0}^{(k \ell)} \\
& -\sum_{k=1}^{K} \overline{\mathbf{f}}^{T} \mathbf{a}^{(k)}
\end{aligned}
$$

in which

$$
\begin{aligned}
\mathbf{H}^{(k)} & =\frac{1}{2} \int_{\Gamma^{(k)}} \mathbf{S}^{(k)} \mathbf{n}^{(k)} \mathbf{U}^{(k)} d \Gamma \\
{ }^{*} \mathbf{h}^{(k)} & =\int_{\Gamma^{(k)}}{ }^{*} \mathbf{N}^{(k)}{ }^{T} \mathbf{n}^{(k)} \mathbf{U}^{(k)}{ }^{T} d \Gamma
\end{aligned}
$$

where $\mathbf{S}^{(k)}$ and $\mathbf{U}^{(k)}$ are given by Eqs. (B31) and (B32). The total potential energy in Eq. (63) is compacted to its final form

$$
\pi=\frac{1}{2} \mathbf{a}^{T} \mathbf{H a}-{ }^{*} \mathbf{h a}+\frac{1}{2} \mathbf{b} \Delta+\tilde{\Lambda}^{T} \tilde{\mathbf{C}} \mathbf{a}-\tilde{\Lambda}^{T} \tilde{\mathbf{f}}+\hat{\Lambda} \hat{\mathbf{C}} \mathbf{a}-\hat{\boldsymbol{\Lambda}}^{T} \hat{\mathbf{g}}_{1} \boldsymbol{\Delta}+\hat{\boldsymbol{\Lambda}}^{T} \hat{\mathbf{g}}_{0}-\overline{\mathbf{f}} \mathbf{a}
$$

in which the vectors and matrices are defined by

$$
\begin{aligned}
& \mathbf{a}^{T}=\left\{\mathbf{a}^{(1)^{T}}, \mathbf{a}^{(2)^{T}}, \ldots, \mathbf{a}^{(K)^{T}}\right\} \\
& \Delta^{T}=\left\{\Delta^{(1)^{T}}, \Delta^{(2)^{T}}, \ldots, \Delta^{(L)}\right\} \\
& \tilde{\Lambda}^{T}=\left\{\tilde{\Lambda}^{(1)}, \tilde{\Lambda}^{(2)^{T}}, \ldots, \tilde{\Lambda}^{(K)^{T}}\right\} \\
& \hat{\Lambda}^{T}=\left\{\hat{\Lambda}^{(1)^{T}}, \hat{\Lambda}^{(2)^{T}}, \ldots, \hat{\Lambda}^{(K)^{T}}\right\} \text { with } \hat{\Lambda}^{(k)^{T}}=\left\{\hat{\Lambda}^{(k 1)^{T}}, \hat{\Lambda}^{(k 2)^{T}}, \ldots, \hat{\Lambda}^{(k L)^{T}}\right\}
\end{aligned}
$$




$$
\begin{aligned}
& \overline{\mathbf{f}}^{T}=\left\{\overline{\mathbf{f}}^{(1)^{T}}, \overline{\mathbf{f}}^{(2)^{T}}, \ldots, \overline{\mathbf{f}}^{(\mathrm{K})^{\mathrm{T}}}\right\} \\
& { }^{*} \mathbf{h}^{T}=\left\{{ }^{*} \mathbf{h}^{(1)^{T}},{ }^{*} \mathbf{h}^{(2)^{T}}, \ldots,{ }^{*} \mathbf{h}^{(\mathrm{K})^{\mathrm{T}}}\right\} \\
& \tilde{\mathbf{f}}^{T}=\left\{\tilde{\mathbf{f}}^{(1)^{T}}, \tilde{\mathbf{f}}^{(2)^{\mathrm{T}}}, \ldots, \tilde{\mathbf{f}}^{(K)^{T}}\right\} \\
& \hat{\mathbf{g}}_{0}^{T}=\left\{\hat{\mathbf{g}}_{0}^{(1)^{T}}, \hat{\mathbf{g}}_{0}^{(2) \mathrm{T}}, \ldots, \hat{\mathbf{g}}_{0}^{(K)^{T}}\right\} \text { with } \hat{\mathbf{g}}_{0}^{(k)^{T}}=\left\{\hat{\mathbf{g}}_{0}^{(k 1)^{T}}, \hat{\mathbf{g}}_{0}^{(k 2)^{T}}, \ldots, \hat{\mathbf{g}}_{0}^{(k L)^{T}}\right\}
\end{aligned}
$$

and

$$
\begin{aligned}
& \mathbf{H}=\left[\begin{array}{llll}
\mathbf{H}^{(1)} & & & \\
& \mathbf{H}^{(2)} & & \\
& & \ddots & \\
& & & \mathbf{H}^{(K)}
\end{array}\right] \\
& \mathbf{b}=\left[\begin{array}{llll}
\mathbf{b}^{(1)} & & & \\
& \mathbf{b}^{(2)} & & \\
& & \ddots & \\
& & & \mathbf{b}^{(L)}
\end{array}\right]
\end{aligned}
$$

$\tilde{\mathbf{C}}=\left[\begin{array}{llll}\tilde{\mathbf{C}}^{(1)} & & & \\ & \tilde{\mathbf{C}}^{(2)} & & \\ & & \ddots & \\ & & & \tilde{\mathbf{C}}^{(K)}\end{array}\right]$

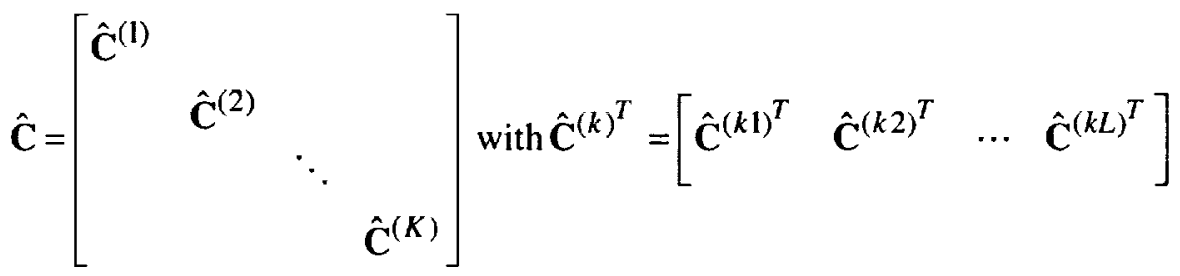

and 


$$
\hat{\mathbf{g}}_{1}^{T}=\left[\begin{array}{llll}
\hat{\mathbf{g}}_{1}^{(1)^{T}} & \hat{\mathbf{g}}_{1}^{(2)^{T}} & \ldots & \hat{\mathbf{g}}_{1}^{(K)^{T}}
\end{array}\right] \text { with } \hat{\mathbf{g}}_{1}^{(k)}=\left[\begin{array}{llll}
\hat{\mathbf{g}}_{1}^{(k 1)} & & & \\
& \hat{\mathbf{g}}_{1}^{(k 2)} & & \\
& & \ddots & \\
& & & \hat{\mathbf{g}}_{1}^{(k L)}
\end{array}\right]
$$

The minimization of the total potential energy, i.e., $\delta \pi=0$, leads to the following equilibrium equations

$$
\left[\begin{array}{cccc}
\mathbf{H} & \mathbf{0} & \tilde{\mathbf{C}}^{T} & \hat{\mathbf{C}}^{T} \\
\mathbf{0} & \mathbf{b} & \mathbf{0} & \hat{\mathbf{g}}_{1}^{T} \\
\tilde{\mathbf{C}} & \mathbf{0} & \mathbf{0} & \mathbf{0} \\
\hat{\mathbf{C}} & \hat{\mathbf{g}}_{1} & \mathbf{0} & \mathbf{0}
\end{array}\right]\left\{\begin{array}{c}
\mathbf{a} \\
\Delta \\
\tilde{\mathbf{\Lambda}} \\
\hat{\mathbf{\Lambda}}
\end{array}\right\}=\left\{\begin{array}{c}
\overline{\mathbf{f}}+{ }^{*} \mathbf{h} \\
\mathbf{0} \\
\tilde{\mathbf{f}} \\
\hat{\mathbf{g}}_{0}
\end{array}\right\}
$$

Solving Eq. (69) for the unknown vectors permits the calculation of the stress and displacement components in each laminate, bolt deflections, and forces exerted by the bolts. Because the angles $\theta_{A}$ and $\theta_{B}$ defining the contact region are unknown, these equations are solved for their assumed values until convergence is achieved through an iterative scheme. This procedure is explained in Appendix D.

\section{NUMERICAL RESULTS}

Three different types of load transfer through bolted joints are considered in order to validate the present analysis. The first configuration is a pin-loaded square plate considered by Ireman et al. (1993). The second configuration is a single-lap joint with four bolts investigated by Xiong and Poon (1994). The third configuration is a joint of dissimilar bars with a single bolt under different uniform temperature changes analyzed previously by Gatewood (1957). Then, the capability of the present analysis is demonstrated by considering two different double-lap joint configurations: one with three bolts under mechanical loading only, uniform temperature change only, and their combination, and the other with seven bolts under mechanical loading only.

A pin-loaded square plate was investigated through detailed finite element analysis by Ireman et al. (1993). As shown in Fig. 5, the geometry of the plate is defined by the parameters $W=24 \mathrm{~mm}, D=6$ $\mathrm{mm}, \delta=0.021 \mathrm{~mm}$, and thickness $t=3.046 \mathrm{~mm}$. Three different sets of laminate material properties considered in the analysis are given in Table 1 . The bolt was assumed to be rigid and the applied load, $P$, was taken as $5483 \mathrm{~N}$. In the present analysis, the number of terms, $N$, retained in the series representation of the complex potential functions, $\Phi_{r}^{(k \ell)}\left(z_{r}^{(k \ell)}\right)$ and $\varphi_{r}^{(k \ell)}\left(z_{r}^{(k \ell)}\right)$, is taken as 80 . The number of terms retained in the series representation of the reaction tractions, $\tilde{\lambda}^{(k)}$ and $\hat{\lambda}^{(k \ell)}$, denoted by $J$ and $I$, 


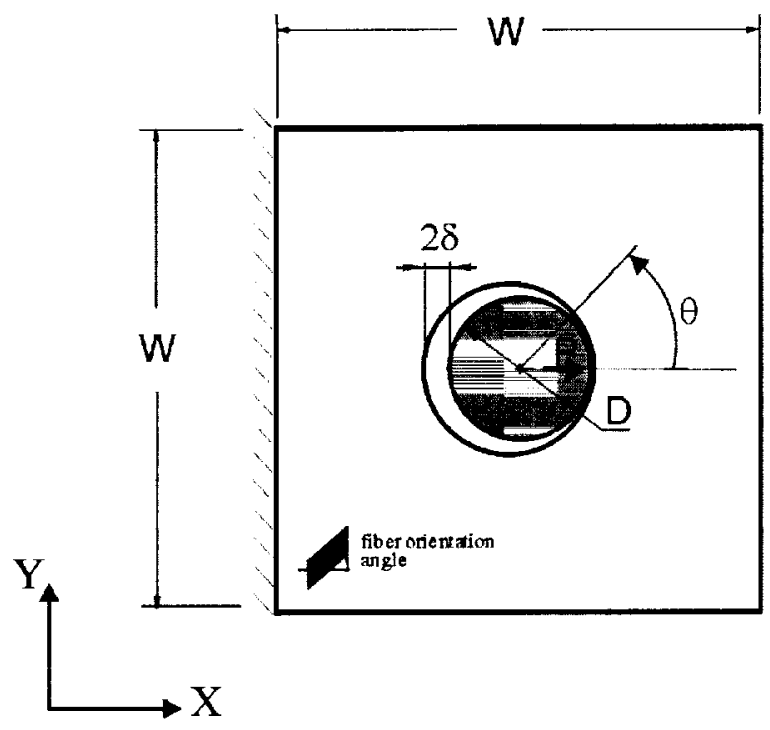

Table 1. Material properties of laminates.

\begin{tabular}{|c|c|c|c|c|}
\hline Case & $E_{x}, \mathrm{GPa}$ & $E_{y}, \mathrm{Gpa}$ & $G_{x y}, \mathrm{GPa}$ & $v_{\mathrm{xy}}$ \\
\hline A & 99.2 & 35.5 & 8.5 & 0.24 \\
\hline B & 35.5 & 99.2 & 8.5 & 0.08 \\
\hline C & 51.5 & 51.5 & 19.3 & 0.33 \\
\hline
\end{tabular}

Fig. 5. Pin-loaded plate configuration.

respectively, is taken as 24 . Figures $6-8$ shows the favorable comparison of the normalized radial and tangential stresses obtained through the finite element analysis reported by Ireman et al. (1993) with those of the present calculations. The radial and tangential stress components are normalized with respect to the applied bearing stress, $-P /(d t)$, and the applied stress, $P /(W t)$, respectively.

The geometry and loading conditions of a single-lap joint of aluminum and composite plates with four bolts are shown in Fig. 9. As shown in this figure, the geometry of the joint is defined by the dimensions of $h^{(1)}=0.31$ in, $h^{(2)}=0.117$ in, $D=0.3125$ in, $W=3.125$ in, $s=1.25$ in, $e=0.9375$ in, $\ell=$ $2.75 \mathrm{in}$. There exists no bolt-hole clearance, $\delta=0$. The composite lay-up is $\left[\left(45^{\circ} / 0^{\circ} /-45^{\circ} / 0^{\circ}\right)_{2} / 0^{\circ} / 90^{\circ}\right]_{S}$ with lamina properties of $E_{L}=18.5 \times 10^{6} \mathrm{psi}, E_{T}=1.9 \times 10^{6} \mathrm{psi}, G_{L T}=0.85 \times 10^{6} \mathrm{psi}$, and $v_{L T}=0.3$. In obtaining the present predictions, the series representations of the functions are truncated at $N=30$ and $J=I=5$. Although the entire geometry of the lap joint is considered in the present analysis, only the predictions for the bolt loads exerted by Bolt 1 and Bolt 3 are presented due to the presence of symmetry. As compared in Table 2, the present analysis predictions are in remarkable agreement with those calculated by Xiong and Poon (1994).

The geometry of the steel and aluminum plates connected with a single bolt is shown in Fig. 10. Each plate is subjected to a different uniform temperature change. The bolt is assumed to be rigid, with no clearance between the bolt and the bolt holes. The material properties and the temperature change for each bar is presented in Table 3. In the present analysis, the bars are assumed as narrow plates whose 


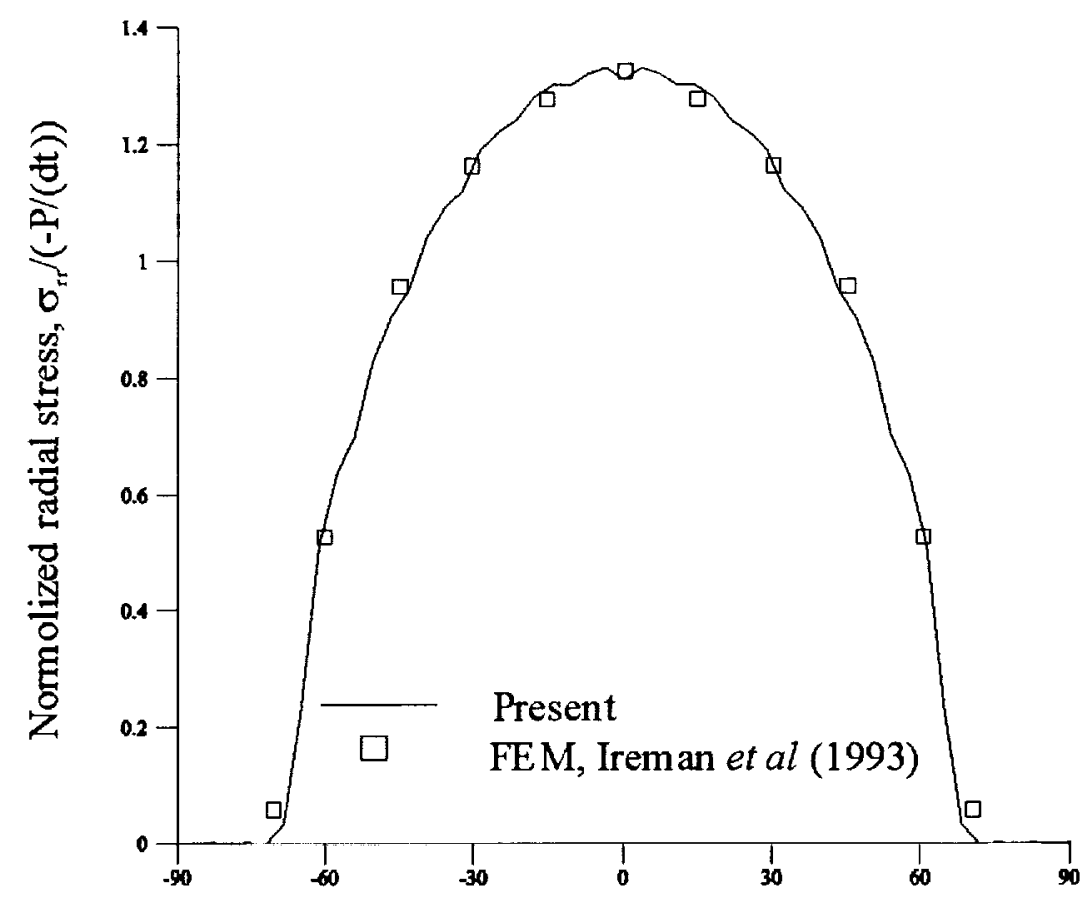

Angle, $\theta$

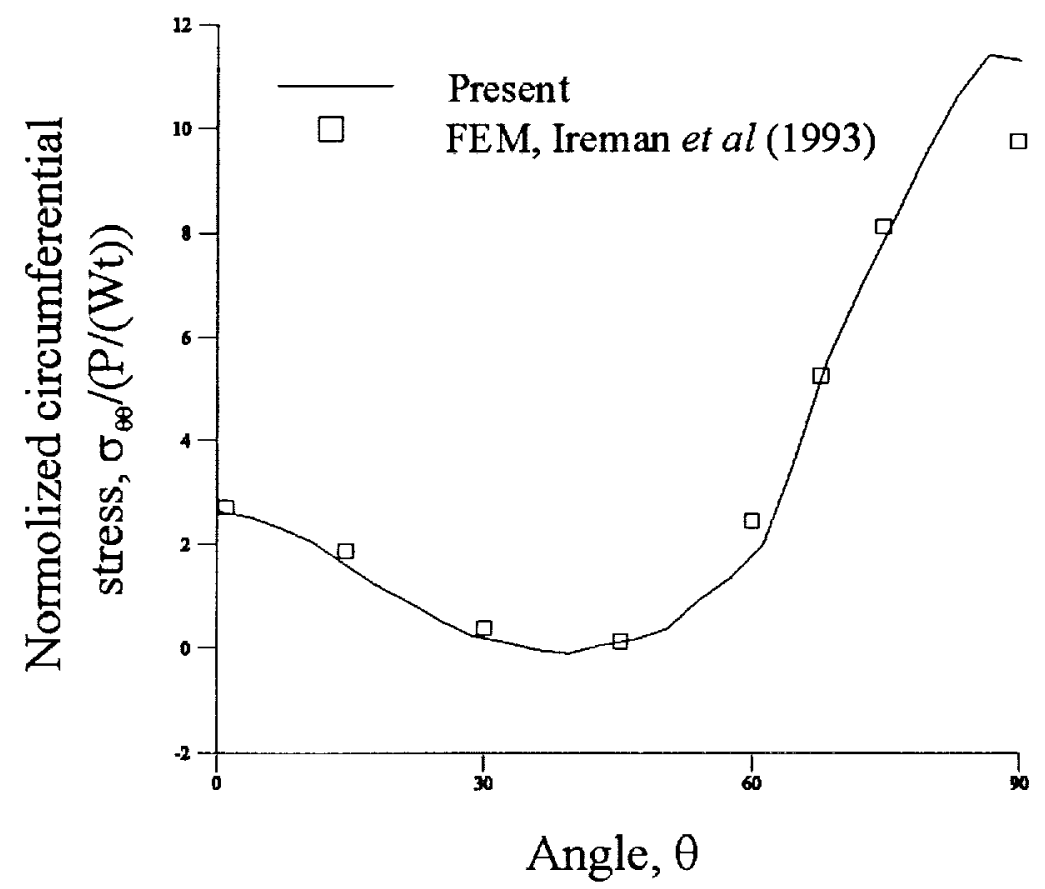

Fig. 6. Comparison of normalized stress around hole for pin-loaded plate for laminate type A. 

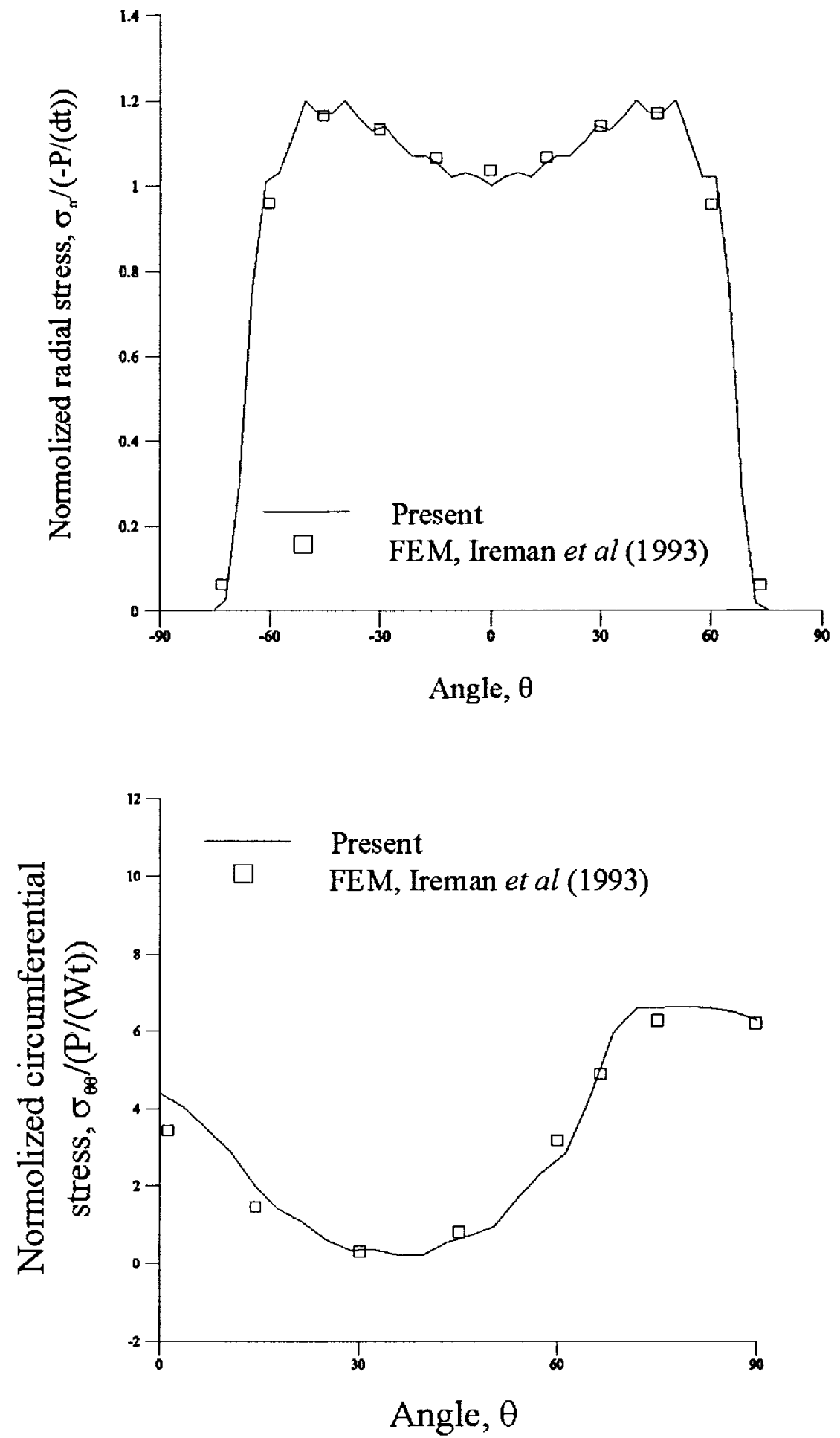

Fig. 7. Comparison of normalized stress around hole for pin-loaded plate for laminate type B. 

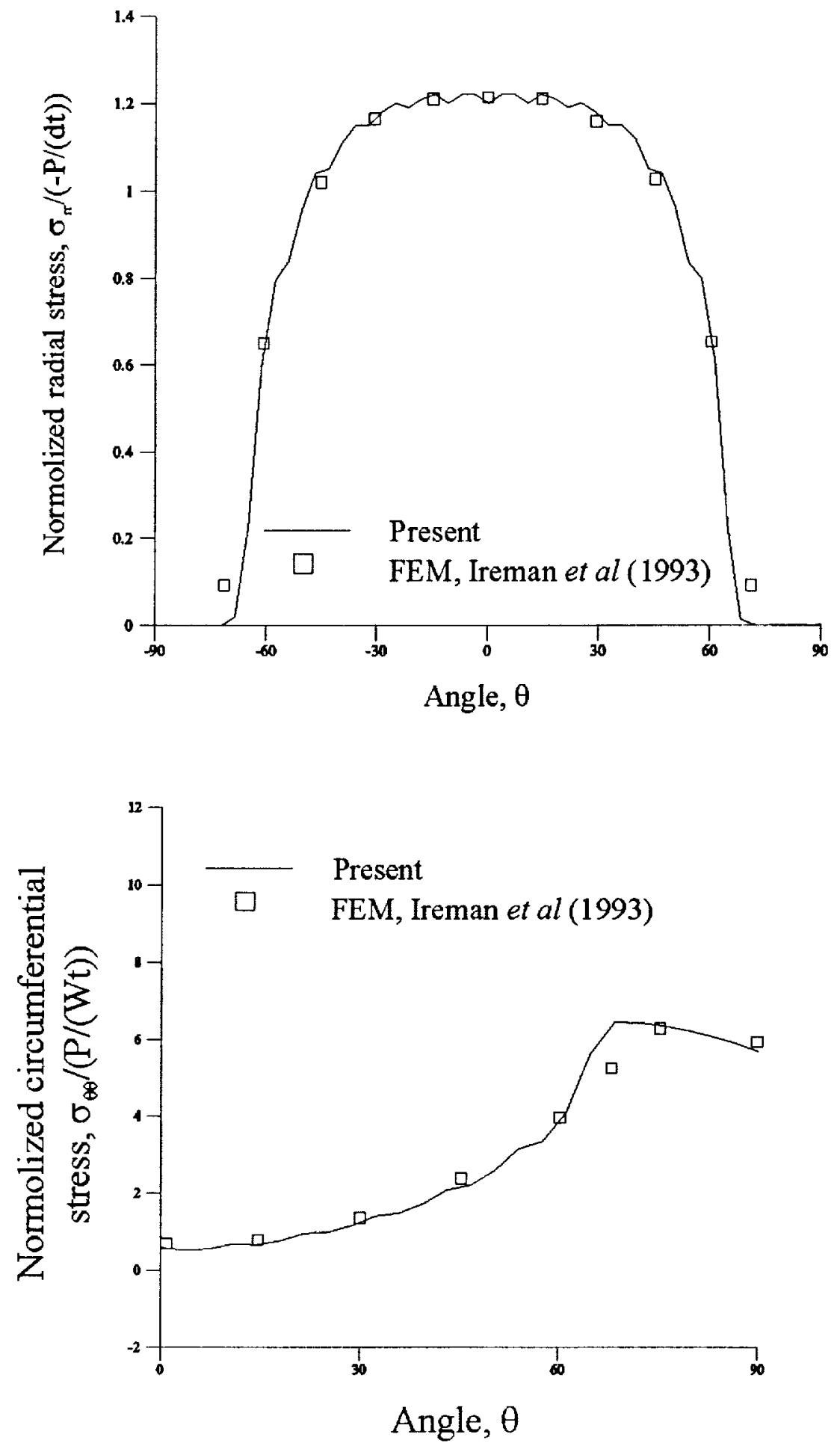

Fig. 8. Comparison of normalized stress around hole for pin-loaded plate for laminate type C. 


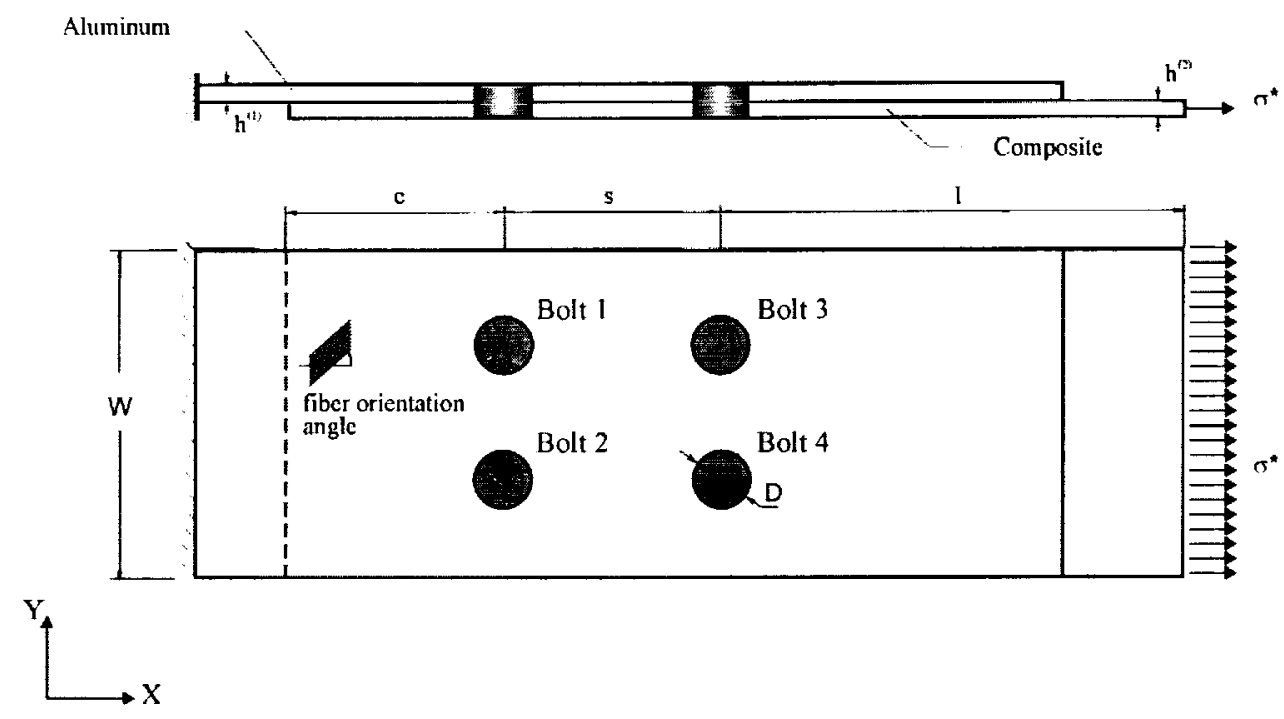

Fig. 9. Single-lap joint with four bolts.

Table 2. Bolt load distribution.

\begin{tabular}{|c|c|c|}
\hline & Present Analysis & Xiong and Poon (1994) \\
\hline$P_{1} / P$ & 0.23 & 0.23 \\
\hline$P_{3} / P$ & 0.27 & 0.27 \\
\hline
\end{tabular}
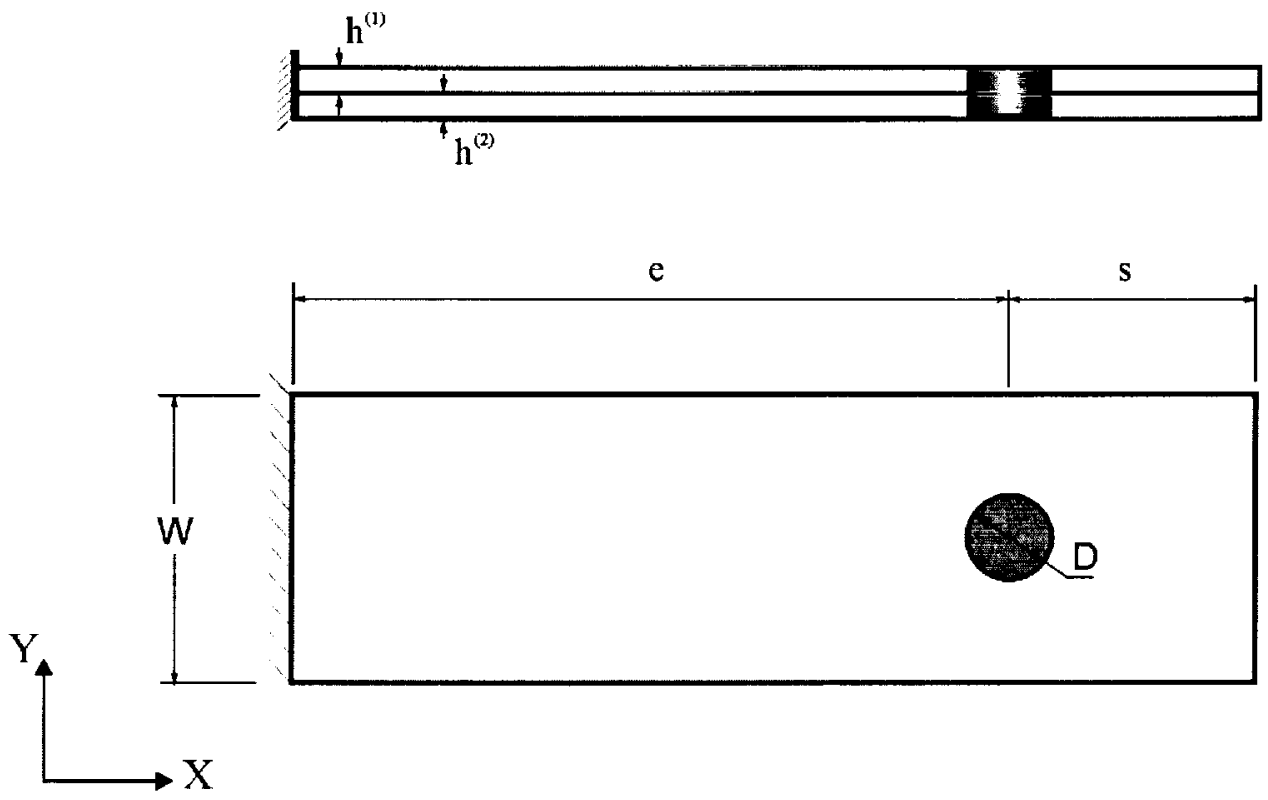

Fig. 10. Steel and Aluminum plates connected with a single bolt under thermal loading. 
Table 3. Material properties and temperature changes.

\begin{tabular}{|c|c|c|c|c|c|}
\hline & Material & $E, \mathrm{psi}$ & $v$ & $\alpha,{ }^{\circ} \mathrm{F}$ & $\Delta T,{ }^{\circ} \mathrm{F}$ \\
\hline Plate 1 & Steel & $3 \times 10^{7}$ & 0.3 & $6.5 \times 10^{-6}$ & 500 \\
\hline Plate 2 & Aluminum & $10^{7}$ & 0.3 & $12 \times 10^{-6}$ & 100 \\
\hline
\end{tabular}

geometry is specified by $W=0.5 \mathrm{in}, e=3.5 \mathrm{in}, s=0.5, D=0.2 \mathrm{in}$, and $h^{(1)}=h^{(2)}=0.06$ in. The present analysis predictions are obtained by considering $N=30$ and $J=I=5$ in the series representations of the functions. The present analysis predicts the force exerted by the bolt to be $442 \mathrm{lb}$. The bolt force obtained by Gatewood (1957) according to the strength of materials approach is $461 \mathrm{lb}$. The difference of $4.1 \%$ in bolt load prediction is possibly due to the fact that the strength of materials approach does not account for the presence of the bolt hole.

The geometry of a double-lap joint with three bolts is shown in Fig. 11. The geometry of the plates all having the same dimensions are described by $W=50 \mathrm{~mm}, s=25 \mathrm{~mm}, e=12.5 \mathrm{~mm}, D=6 \mathrm{~mm}, h=5$ $\mathrm{mm}, h^{(1)}=h^{(2)}=4 \mathrm{~mm}$, and $\delta=0.005 \mathrm{~mm}$. The material properties for the steel and aluminum plates, respectively, are: $E_{S}=200 \mathrm{GPa}, v_{S}=0.3, \alpha_{S}=11.7 \times 10^{-6} /{ }^{\circ} \mathrm{F}, E_{A}=70 \mathrm{GPa}, v_{A}=0.3$, and $\alpha_{A}=23.0 \times 10^{-6}$ $I^{\circ} \mathrm{F}$. All of the bolts in this joint are assumed to be rigid. For the first case of mechanical loading only, the applied tensile stress, $\sigma^{*}$, is $200 \mathrm{~N} / \mathrm{mm}$. In the second loading case, the plates are under uniform temperature change only. Accordingly, the steel plates are free from temperature change, $\Delta T_{S}=0^{\circ} \mathrm{C}$, while the aluminum plate is subject to $\Delta T_{A}=125^{\circ} \mathrm{C}$. The third case represents a combination of the mechanical and thermal loads, with $\sigma^{*}=200 \mathrm{~N} / \mathrm{mm}, \Delta T_{S}=0^{\circ} \mathrm{C}$, and $\Delta T_{A}=125^{\circ} \mathrm{C}$. The number of terms retained in the series representation of the functions in the present analysis is specified by $N=30$ and $J=I=5$.

Due to the presence of symmetry, only the results concerning Bolt 2 and Bolt 3 are presented in Figs. 12-14 and Table 4. The tangential and radial stresses around the bolt holes, shown in Figs. 12-14, correspond to mechanical loading, uniform temperature change, and their combination, respectively. As observed in these figures, the location and extent of the contact region, as well as the magnitudes of the bearing stresses, change significantly. The bolt load distributions for each of these loading cases are given in Table 4. As expected, the force exerted by Bolt 2 alters its direction when the nature of the loading changes from mechanical to thermal. This behavior is caused by the thermal expansion of the aluminum plate constrained with three bolts. Consequently, under thermal loading, the bolt forces with their directions toward the middle of the plate resist the thermal expansion of the plate. 


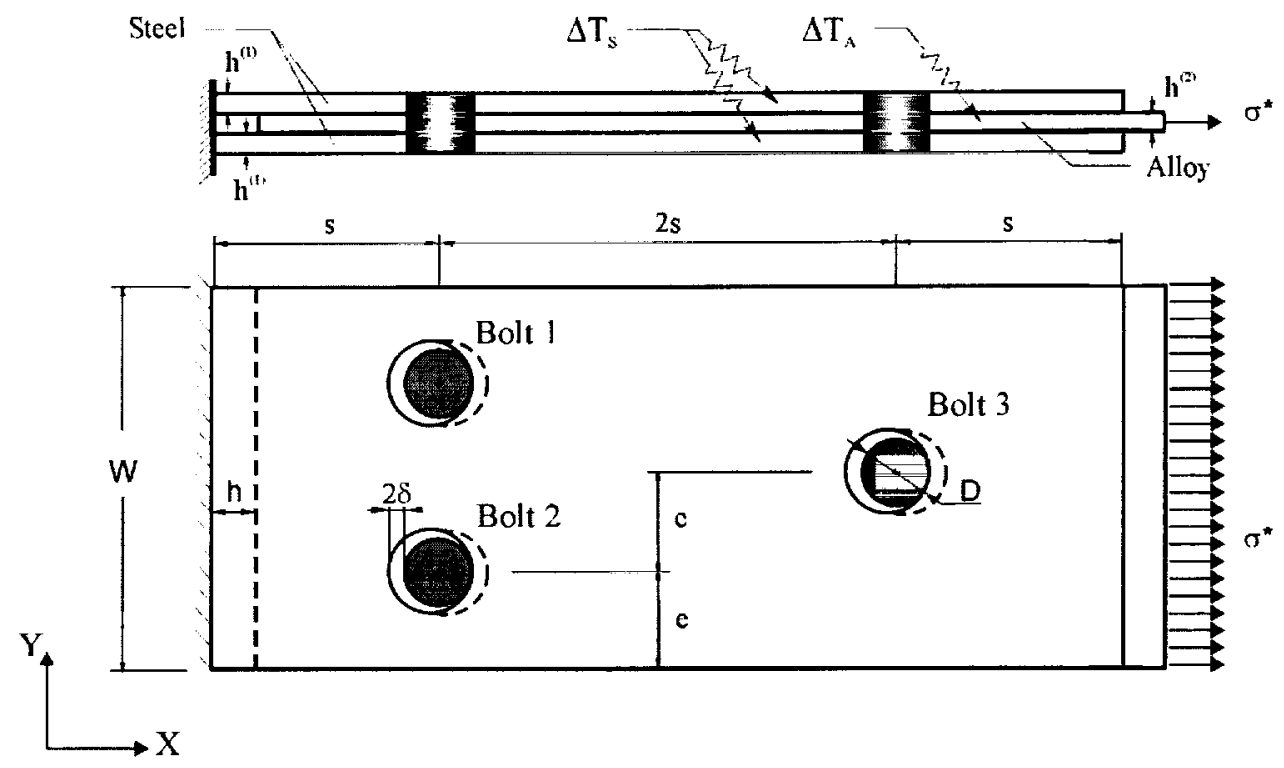

Fig. 11. Steel plates bolted to an aluminum plate with three bolts.

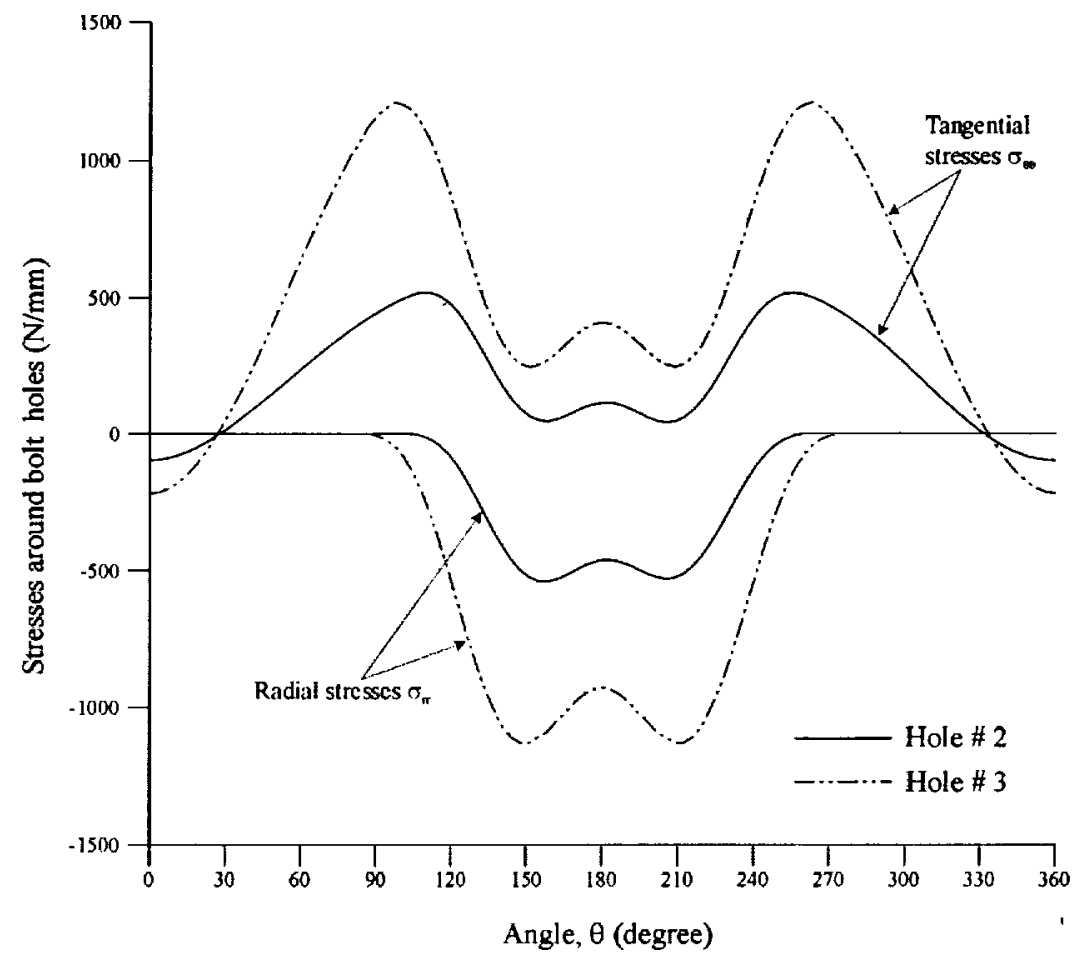

Fig. 12.Stresses around bolt holes in alloy plate due to mechanical loading only. 


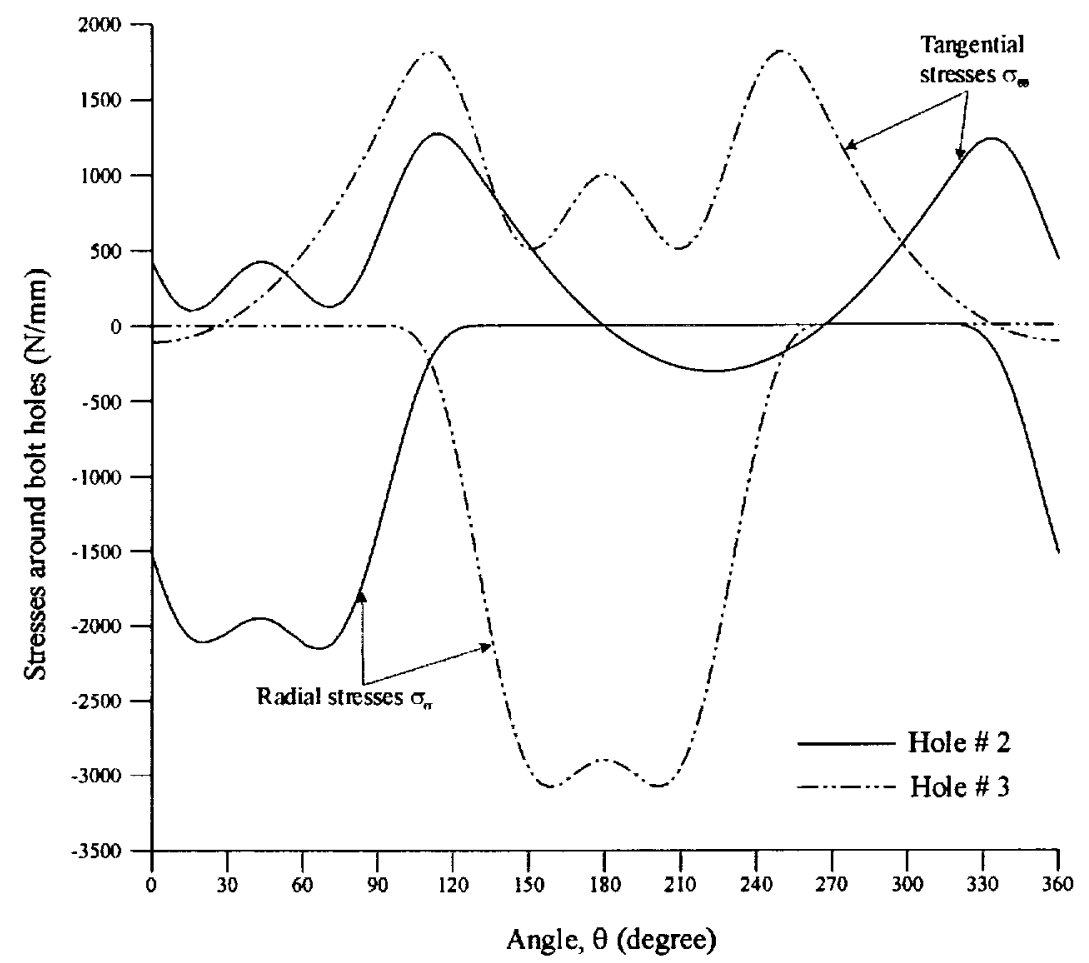

Fig. 13. Stresses around bolt holes in alloy plate due to thermal loading only.

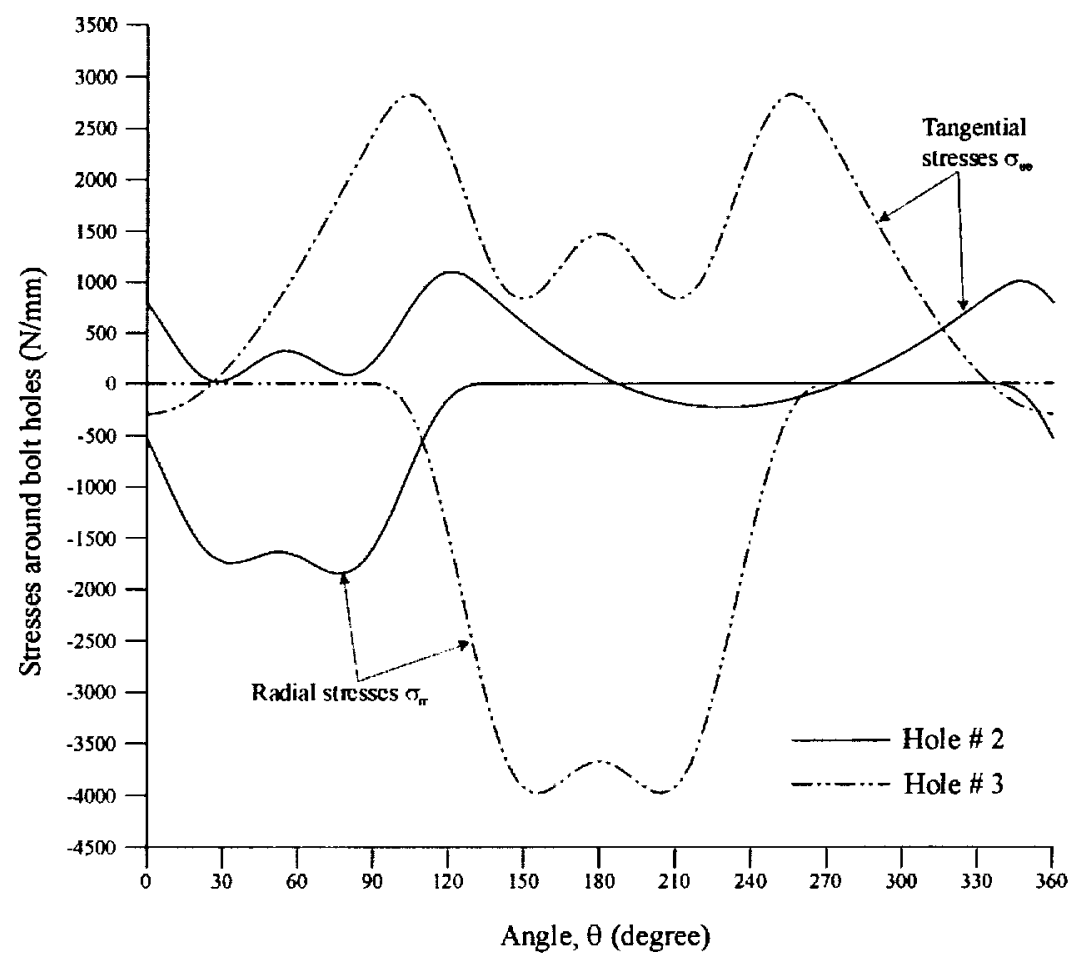

Fig. 14. Stresses around bolt holes in alloy plate due to combined thermal-mechanical loading. 
Table 4. Bolt load distribution in a double-lap joint of steel plates and an aluminum plate with three bolts.

\begin{tabular}{|c|c|c|c|c|}
\hline & & \multicolumn{3}{|c|}{ Bolt load, $N$} \\
\cline { 3 - 5 } & & Mechanical Loading & Thermal Loading & Combined Loading \\
\hline \multirow{2}{*}{ Bolt \# 2 } & $F_{x}$ & -2313.99 & 6906.94 & 4383.20 \\
\cline { 2 - 5 } & $F_{y}$ & -54.74 & 6597.65 & 6372.89 \\
\hline \multirow{2}{*}{ Bolt \# 3 } & $F_{x}$ & -5374.18 & -13815.46 & -18769.19 \\
\cline { 2 - 5 } & $F_{y}$ & 0.00 & 0.00 & 0.00 \\
\hline
\end{tabular}

Under combined mechanical and thermal loading, the forces exerted by Bolt 2 and Bolt 3 are approximately equal to the sum of the forces acting on these bolts in the cases of mechanical and thermal loads. This shows the effect of the non-linearity arising from the contact analysis.

A complex double-lap joint with seven bolts, shown in Fig. 15, is subjected to a tensile loading. The outer plates are steel and the inner plate is a composite. The bolt-hole clearance is specified as $1 \%$ of the hole diameter. The composite plate is made of graphite and fiberglass with material properties $E_{x}=4.7 \times 10^{6} \mathrm{psi}, E_{T}=4.75 \times 10^{6} \mathrm{psi}, G_{L T}=1.2 \times 10^{6} \mathrm{psi}$, and $v_{x y}=0.24$. Steel properties for the plates and bolts are taken as $E=30 \cdot 10^{6} \mathrm{psi}$ and $v=0.3$. The applied load is $70,000 \mathrm{lbs}$, corresponding to $\sigma^{*}=10,000$ psi.

In the calculation of the results, the number of terms retained in the series representation of the functions in the present analysis is specified by $N=30$ and $J=I=7$. Because of the presence of symmetry, only the stress distributions around Bolts 1, 2, 4, and 5 are shown in Fig. 16. The forces exerted by these bolts are given in Table 5. As expected, the bolts in the first row share more of the load than the bolts in the second row. Also, the outer bolts share more of the load than the inner bolts. Accordingly, Bolt 1 and Bolt 3 are the most highly loaded bolts for this configuration.

\section{CONCLUSIONS}

In this study, a new approach based on a complex potential theory in conjunction with a variational formulation has been introduced for the thermo-elastic contact analysis of a general bolted-joint configuration containing multiple laminates joined by multiple bolts. The total potential energy of the joint is formulated by using a solution in the form of a complex potential series that automatically satisfies the stress equilibrium equations and compatibility conditions, thus avoiding the necessity to perform area integrations and resulting in boundary integral expressions for the strain energy of the laminates. The total potential energy also includes the strain energy of the bolts based on a shear deformable beam theory. 


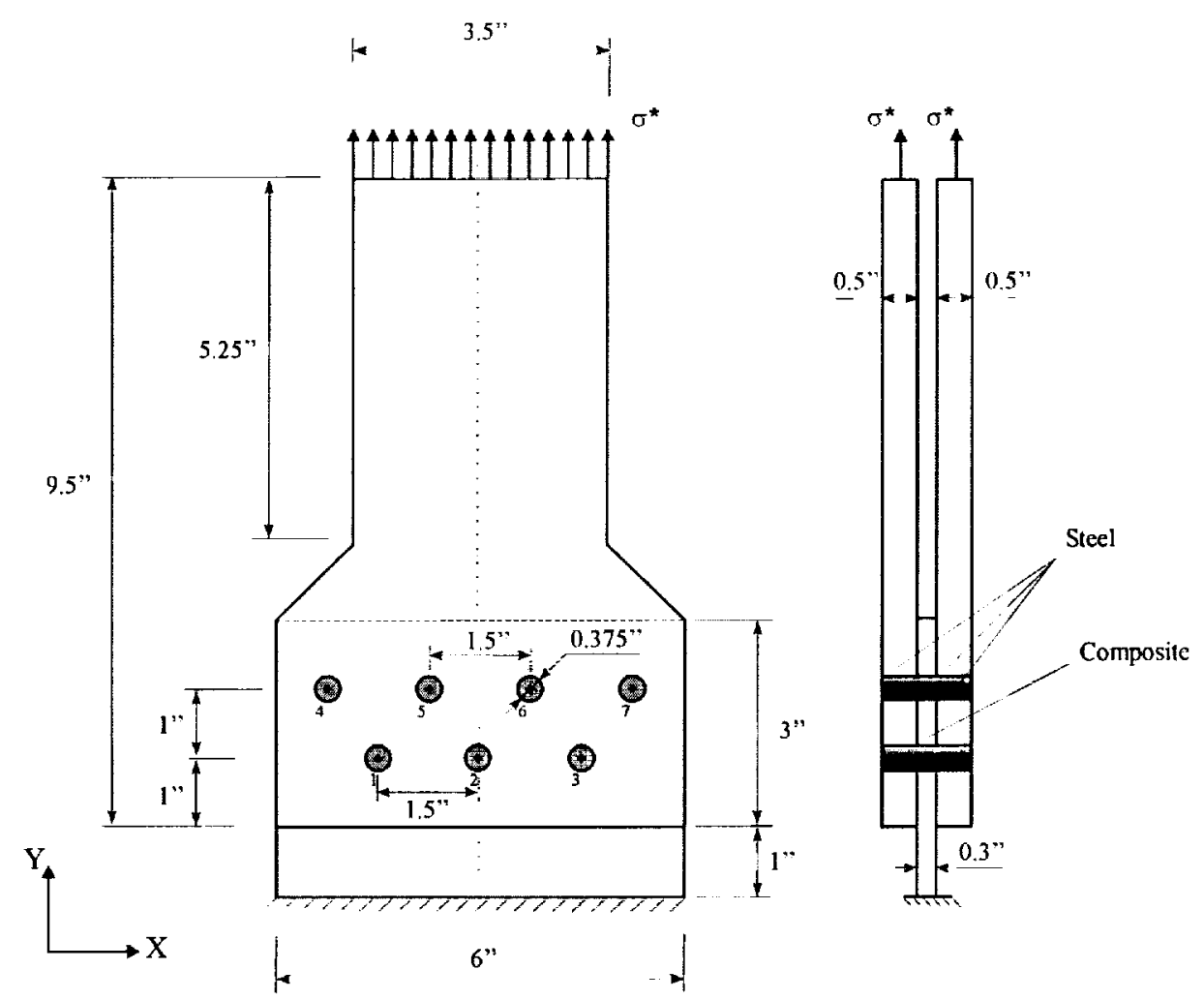

Fig. 15. Steel plates bolted to a composite laminate with seven bolts.

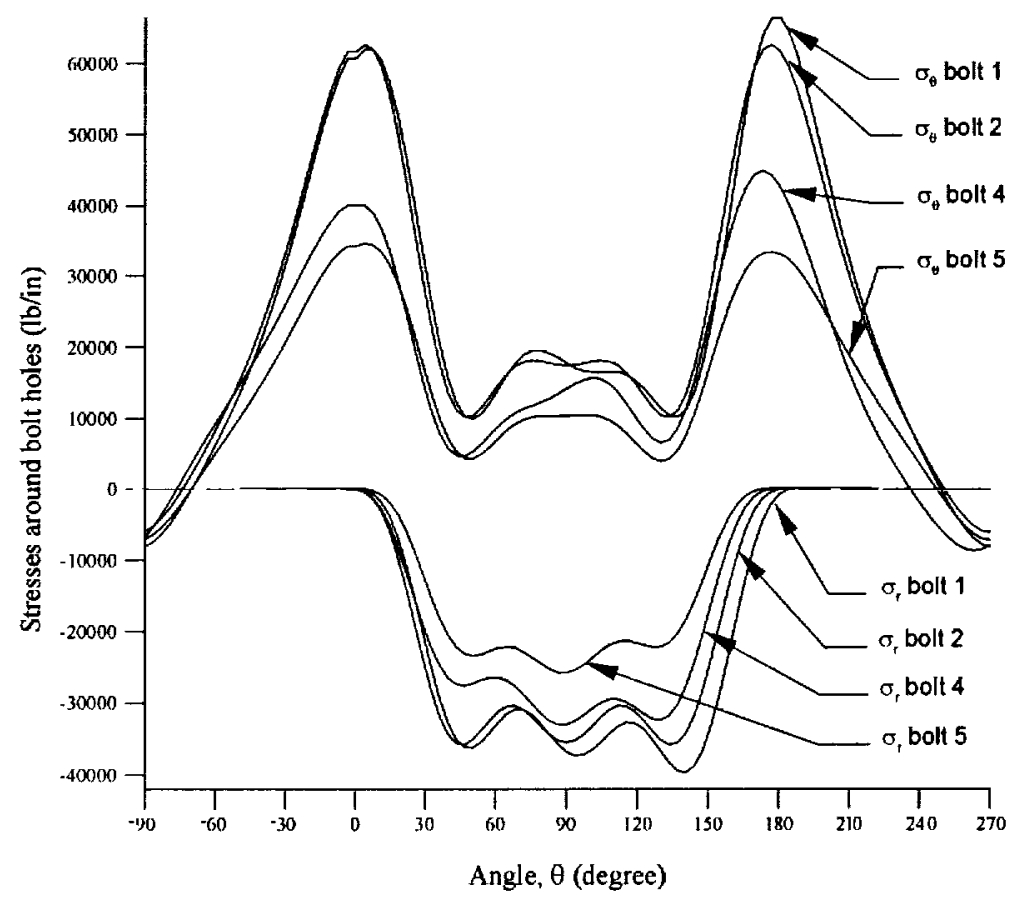

Fig. 16. Stresses around bolt holes in composite plate with seven bolts. 
Table 5. Bolt load distribution in a double-lap joint of steel plates and a composite laminate with seven bolts.

\begin{tabular}{|c|c|c|l|}
\hline Bolt & $F_{x}, \mathrm{lb}$ & $F_{y}, \mathrm{lb}$ & $F_{y} / F_{\text {applied }}$ \\
\hline 1 & -1181.5 & 11983.7 & 0.1712 \\
\hline 2 & 0.0 & 11340.9 & 0.1620 \\
\hline 4 & 2.5 & 9947.8 & 0.1421 \\
\hline 5 & 238.3 & 7427.6 & 0.1061 \\
\hline
\end{tabular}

In order to capture high gradient variations of stresses near the free or bolted holes, the stress field is defined as the superposition of complex potential series originating from each hole. Hence, not only are continuous stress and displacement fields obtained but the modeling of the entire joint is also simplified considerably. By only entering boundary information, hole size and locations, and the number of terms to be used in complex and other series, the solution provides all the stress, displacement, and contact force distributions at any point in the joint.

Contact between the bolts and the laminates is established by enforcing displacement continuity along the contact region between the bolts and the plates. This is established by incorporating the work done by the unknown contact forces over the contact displacements into the total potential energy expression. The contact displacements are defined by constraint equations that take into account the gap between the bolts and plates. The contact forces are assumed in the form of trigonometric series that satisfy stress-free conditions at the ends of the contact regions.

Since the contact regions are unknown a priori, an iterative scheme is adopted in order to determine the beginning and end angles of the contact regions. Starting with an initial guess, the system matrix is generated to solve for unknown plate and bolt displacements and contact forces simultaneously. The simultaneous determination of bolt displacements and contact forces, along with the plate displacements, is a unique feature of the present formulation. A new guess is then obtained by monitoring the stress distribution along the contact regions. The iterative scheme is continued until a configuration for contact regions is reached where all the contact forces become compressive.

The validation problems show excellent agreement of the present formulation against those reported by other investigators. The pin-loaded panel in the first validation problem provides a comparison of contact angles and contact force distribution. For all laminate configurations, remarkable agreement is obtained between the present analysis and the refined finite element solution. In the case of a single-lap configuration subjected to thermal loading, the strength of material solution is available. As expected, the present analysis achieves the right bolt load and compares well with the strength of material solution if 
full contact is assumed around the bolt. In the case of a single- and a double-lap joint containing multiple holes, the present analysis captures the correct load distribution shared by each bolt.

The versatility of the present formulation has been demonstrated by solving a double-lap joint configuration containing three bolts and two laminates with different material properties, and the joint is subjected to thermal, mechanical, and thermo-mechanical loadings. All of these cases are solved assuming variable contact regions. Therefore, the rule of superposition is invalid for these problems since the contact regions are changing. This can be clearly observed in the tabulated results where the summation of the bolt load distribution arising from thermal and mechanical loads is significantly different from those arising from the combined loading.

\section{REFERENCES}

Bowie, O. L. 1956, "Analysis of an Infinite Plate Containing Radial Cracks Originating at the Boundary of an Internal Circular Hole," Journal of Mathematics and Physics, Vol. 35, pp. 60-71.

Gatewood, B. E., 1957, Thermal Stresses, McGraw-Hill Book Company, Inc., New York.

Ireman, T., Nyman, T., and Hellbom, K., 1993, "On Design Methods for Bolted Joints in Composite Aircraft Structures," Composite Structures, Vol. 25, pp. 567-578.

Lekhnitskii, S. G., 1968, Anisotropic Plates, Gordon and Breach Science Publishers, Inc., New York.

Xiong, Y. and Poon, C., 1994, "A Design Model for Composite Joints with Multiple Fasteners," Aeronautical Note IAR-AN-80, NRC No. 3216, National Research Council, Canada.

Madenci, E., Shkarayev, S., and Sergeev, B., 1997, "Analysis of Composite Laminates with Multiple Fasteners," Report to The Federal Aviation Administration, University of Arizona, Tucson, AZ.

Madenci, E., Shkarayev, S., Sergeev, B., Oplinger, D. W., and Shyprykevich, P., 1998, "Analysis of Composite Laminates with Multiple Fasteners, International Journal of Solids and Structures, Vol. 35, pp. 1793-1811.

Madenci, E., Sergeev, B., Shipilov, Y., and Scotti, S., 1999, “Analysis of Double-Lap Composite Joints with Multiple-Row Bolts Under Combined Mechanical and Thermal Loading," International Conference on Joining and Repair of Plastics and Composites, The Institution of Mechanical Engineers, London, England, pp. 55-64, 1999. 


\section{APPENDIX A}

The bolt stiffness matrix is derived based on the Timoshenko's zeroth-order shear deformable beam theory. The cross-section of a bolt connecting $K$ laminates (regions) is shown in Fig. Al. The bolt number is denoted by $\ell$ and the regions are numbered from bottom to top in sequential order. The bolt has a circular uniform cross-section, $A_{\ell}$, moment of inertia, $I_{\ell}$, and Young's and shear moduli, $E_{\ell}$ and $G_{\ell}$, respectively. The nuts at the ends of the bolt are assumed to represent clamped boundary conditions, thus preventing rotations but creating reaction moments at the ends of the bolt.

The bolt is subjected to forces arising from the contact between the bolt and the laminates. Because of the variation in laminate thickness and stiffness, these forces exerted by the laminates vary through the length of the bolt. Because of the variable contact forces along the length of the bolt, the large in-plane bolt stiffness compared to those of the laminates and the small ratio of the bolt diameter to its length, the most suitable and accurate representation of the bolt can be achieved by discretizing the bolt into small Timoshenko beam elements connected at nodal points, as shown in Fig. Al.

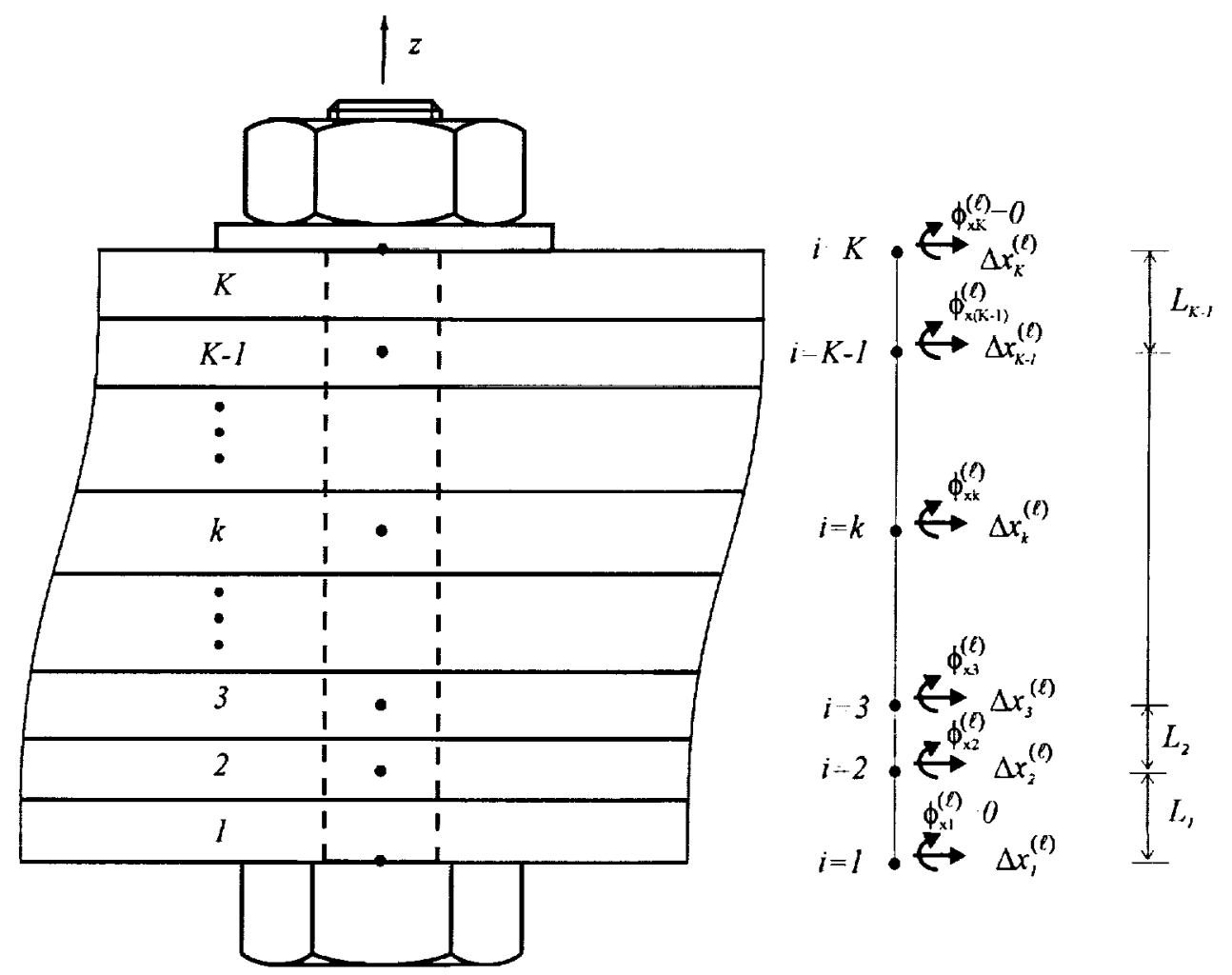

Fig. Al. A close view of the section in the vicinity of a bolt, and the discretization of the bolt into Timoshenko beam elements. 
The bolt discretization is based on the most effective locations of the contact forces. Thus, two of the nodes are selected at the top and bottom ends of the bolt in order to obtain the largest in-plane bolt deflection. The contact forces exerted on the bolt by the top and bottom laminates are assigned to these end nodal points. The remaining nodal points are chosen at the intersections of mid-planes of the inner laminates and the bolt longitudinal axis, as shown in Fig. Al. Hence, the contact forces exerted on the bolt by the inner laminates are assigned to these intermediate nodal points. The nodal deflections and rotations along the length of the bolt permit the determination of the bolt deflection at any point along the bolt by utilizing interpolation functions.

Because the bolt material is homogeneous and isotropic and its cross-section is circular, the moment of inertia of the bolt is homogeneous on the $(x, y)$ plane. Hence, there exists no coupling between the deformations of the bolt on the $(x, z)$ and $(y, z)$ planes, leading to the uncoupled stiffness matrices of the bolt associated with the $(x, z)$ and $(y, z)$ planes. However, their forms are identical since the stiffness properties of the bolt are homogeneous on the $(x, y)$ plane. Therefore, the derivation of the stiffness matrix associated with the $(x, z)$ plane applies to the stiffness matrix associated with the $(y, z)$ plane.

As shown in Fig. Al, the bolt connecting $K$ laminates is modeled by $(K-1)$ number of beam elements and $K$ nodes. Each node is assigned a deflection, $\Delta_{x i}^{(\ell)}=\Delta_{x}^{(\ell)}\left(z_{i}\right)$, and a rotation, $\phi_{x i}^{(\ell)}=\phi_{x}^{(\ell)}\left(z_{i}\right)$, with the subscript $i=1, K$ representing the node numbers. The positive directions of the deflections and rotations are shown in Fig. A1. Also, the length of each beam element is denoted by $L_{i}$, with $i=1, K$. Based on the geometry and material properties of the $\ell^{\text {th }}$ bolt, the strain energy arising from the bolt deformation associated with the $(x, z)$ plane can be written as

$$
U_{x}^{(\ell)}=\sum_{i=1}^{K-1} U_{x}^{(i \ell)}
$$

The strain energy of each element is expressed as

$$
U_{x}^{(i \ell)}=\frac{1}{2} \int_{L_{i}} E_{\ell} I_{\ell}\left(\kappa_{z z}^{(i \ell)}\right)^{2} d z+\frac{1}{2} \int_{L_{i}} G_{\ell} A_{f \ell}\left(\gamma_{z x}^{(i \ell)}\right)^{2} d z
$$

in which the strains $\kappa_{z z}^{(i \ell)}$ and $\gamma_{z x}^{(i \ell)}$ are based on Timoshenko's zeroth-order shear deformation theory, and they are defined as

$$
\kappa_{z z}^{(i \ell)}=-\frac{d^{2} \Delta_{x}^{(i \ell)}}{d z^{2}} \text { and } \gamma_{z x}^{(i \ell)}=\frac{d \Delta_{x}^{(i \ell)}}{d z}-\phi_{x}^{(i \ell)}
$$

In Eq. (A2), $A_{f \ell}$ denotes the corrected area of the bolt and is defined as 


$$
A_{f \ell}=c^{2} A_{\ell}
$$

in which $c^{2}$ represents the shear correction factor, which is a correction to the strain energy due to uniform transverse shear deformations.

The displacement and rotation field of the bolt is represented by piecewise continuous interpolation functions. These functions are defined individually over each element as

$$
\begin{gathered}
\Delta_{x}^{(i \ell)}(z)=H_{1}(s) \Delta_{x i}^{(\ell)}+H_{2}(s) \Delta_{x(i+1)}^{(\ell)}+H_{3}(s) \frac{d \Delta_{x i}^{(\ell)}}{d z}+H_{4}(s) \frac{d \Delta_{x(i+1)}^{(\ell)}}{d z} \\
\phi_{x}^{(i \ell)}(z)=N_{1}(s) \phi_{x i}^{(\ell)}+N_{2}(s) \phi_{x(i+1)}^{(\ell)}+N_{3}(s) \phi_{x m}^{(\ell)}
\end{gathered}
$$

where the subscript $m$ denotes the mid-point of the element. The variable $s=z-z_{i}$ is a local coordinate system for the $i^{\text {th }}$ element. The interpolation functions $H_{j}(s)(j=1, \ldots, 4)$ represent the cubic Hermitian polynomials defined as

$$
\begin{aligned}
& H_{1}(s)=1-\frac{3 s^{2}}{L_{i}^{2}}+\frac{2 s^{3}}{L_{i}^{3}} \\
& H_{2}(s)=\frac{3 s^{2}}{L_{i}^{2}}-\frac{2 s^{3}}{L_{i}^{3}} \\
& H_{3}(s)=s-\frac{2 s^{2}}{L_{i}}+\frac{s^{3}}{L_{i}^{2}} \\
& H_{4}(s)=-\frac{s^{2}}{L_{i}}+\frac{s^{3}}{L_{i}^{2}}
\end{aligned}
$$

Also, the functions $N_{j}(s)(j=1, . ., 3)$ represent the Lagrangian shape functions defined as

$$
\begin{gathered}
N_{1}(s)=\left(s-L_{i} / 2\right)\left(s-L_{i}\right) /\left[L_{i}^{2} / 2\right] \\
N_{2}(s)=s\left(s-L_{i}\right) /\left[L_{i}^{2} / 2\right] \\
N_{3}(s)=s\left(L_{i}-s\right) /\left[L_{i}^{2} / 4\right]
\end{gathered}
$$

In order to express $\Delta_{x}^{(i \ell)}$ and $\phi_{x}^{(i \ell)}$ in terms of the nodal unknowns defined at the end points of the element, two successive steps are performed. In the first step, constraint of uniform shearing strain along the beam element is enforced into the kinematic field, thus leading to 


$$
\frac{d \gamma_{z x}^{(i \ell)}}{d z}=\frac{d^{2} \Delta_{x}^{(i \ell)}}{d z^{2}}-\frac{d \phi_{x}^{(i \ell)}}{d z}=0
$$

Substituting from Eqs. (A3) and (A5) into Eq. (A8) and grouping the terms as coefficients of $z^{0}$ and $z$, this constraint equation produces two algebraic equations of the form

$$
\begin{aligned}
& \frac{4}{L_{i}} \frac{d \Delta_{x i}^{(\ell)}}{d z}+\frac{2}{L_{i}} \frac{d \Delta_{x i+1}^{(\ell)}}{d z}=-\frac{6}{L_{i}^{2}} \Delta_{x i}^{(\ell)}+\frac{6}{L_{i}^{2}} \Delta_{x(i+1)}^{(\ell)}+\frac{3}{L_{i}} \phi_{x i}^{(\ell)}+\frac{1}{L_{i}} \phi_{x(i+1)}^{(\ell)}-\frac{4}{L_{i}} \phi_{x m}^{(\ell)} \\
& \frac{6}{L_{i}^{2}} \frac{d \Delta_{x i}^{(\ell)}}{d z}+\frac{6}{L_{i}^{2}} \frac{d \Delta_{x i+1}^{(\ell)}}{d z}=-\frac{12}{L_{i}^{3}} \Delta_{x i}^{(\ell)}+\frac{12}{L_{i}^{3}} \Delta_{x(i+1)}^{(\ell)}+\frac{4}{L_{i}^{2}} \phi_{x i}^{(\ell)}+\frac{4}{L_{i}^{2}} \phi_{x(i+1)}^{(\ell)}-\frac{8}{L_{i}^{2}} \phi_{x m}^{(\ell)}
\end{aligned}
$$

These two equations are then solved for $d \Delta_{x i}^{(\ell)} / d z$ and $d \Delta_{x(i+1)}^{(\ell)} / d z$ as

$$
\begin{gathered}
\frac{d \Delta_{x i}^{(\ell)}}{d z}=-\frac{1}{L_{i}} \Delta_{x i}^{(\ell)}+\frac{1}{L_{i}} \Delta_{x(i+1)}^{(\ell)}+\frac{5}{6} \phi_{x i}^{(\ell)}-\frac{1}{6} \phi_{x(i+1)}^{(\ell)}-\frac{2}{3} \phi_{x m}^{(\ell)} \\
\frac{d \Delta_{x(i+1)}^{(\ell)}}{d z}=-\frac{1}{L_{i}} \Delta_{x i}^{(\ell)}+\frac{1}{L_{i}} \Delta_{x(i+1)}^{(\ell)}-\frac{1}{6} \phi_{x i}^{(\ell)}+\frac{5}{6} \phi_{x(i+1)}^{(\ell)}-\frac{2}{3} \phi_{x m}^{(\ell)}
\end{gathered}
$$

Substituting from Eq. (A10) into Eq. (A5a) and rearranging the terms, the in-plane deflection component, $\Delta_{x}^{(i \ell)}$, is obtained as

$$
\begin{aligned}
\Delta_{x}^{(i \ell)} & =\left(1-\frac{s}{L_{i}}\right) \Delta_{x i}^{(\ell)}+\frac{s}{L_{i}} \Delta_{x(i+1)}^{(\ell)}+\left(\frac{5 s}{6}-\frac{3 s^{2}}{2 L_{i}}+\frac{2 s^{3}}{3 L_{i}^{2}}\right) \phi_{x i}^{(\ell)} \\
& +\left(-\frac{s}{6}-\frac{s^{2}}{2 L_{i}}+\frac{2 s^{3}}{3 L_{i}^{2}}\right) \phi_{x(i+1)}^{(\ell)}+\left(-\frac{2 s}{6}+\frac{2 s^{2}}{L_{i}}-\frac{4 s^{3}}{3 L_{i}^{2}}\right) \phi_{x m}^{(\ell)}
\end{aligned}
$$

Thus, the total number of unknowns in the expressions for the deflection and rotation is reduced from 7 to 5. Substituting from Eqs. (Al1) and (A5) for $\Delta_{x}^{(i \ell)}$ and $\phi_{x}^{(i \ell)}$ in the strain definitions, Eq. (A3), and carrying out the integrations in Eq. (A2), analytically result in the following strain energy expression for the $i^{\text {th }}$ element of the $\ell^{\text {th }}$ bolt in matrix form

$$
U_{x}^{(i \ell)}=\frac{1}{2}\left\{\begin{array}{l}
\mathbf{q}_{x 1}^{(i \ell)} \\
q_{x 2}^{(i \ell)}
\end{array}\right\}^{T}\left[\begin{array}{cc}
\mathbf{b}_{11}^{(i \ell)} & \mathbf{b}_{12}^{(i \ell)} \\
\mathbf{b}_{12}^{(i \ell)} & b_{22}^{(i \ell)}
\end{array}\right]\left\{\begin{array}{c}
\mathbf{q}_{x 1}^{(i \ell)} \\
q_{x 2}^{(i \ell)}
\end{array}\right\}
$$

where 


$$
\begin{aligned}
& \mathbf{q}_{x 1}^{(i \ell)}=\left\{\begin{array}{llll}
\Delta_{x i}^{(\ell)} & \Delta_{x(i+1)}^{(\ell)} & \phi_{x i}^{(\ell)} & \phi_{x(i+1)}^{(\ell)}
\end{array}\right\} \\
& q_{x 2}^{(i \ell)}=\phi_{x m}^{(\ell)} \\
& \mathbf{b}_{11}^{(i \ell)}=\left[\begin{array}{cccc}
\frac{G_{\ell} A_{f \ell}}{L_{i}} & -\frac{G_{\ell} A_{f \ell}}{L_{i}} & \frac{G_{\ell} A_{f \ell}}{6} & \frac{G_{\ell} A_{f \ell}}{6} \\
-\frac{G_{\ell} A_{f \ell}}{L_{i}} & \frac{G_{\ell} A_{f \ell}}{L_{i}} & -\frac{G_{\ell} A_{f \ell}}{6} & \frac{G_{\ell} A_{f \ell}}{6} \\
\frac{G_{\ell} A_{f \ell}}{L_{i}} & -\frac{G_{\ell} A_{f \ell}}{L_{i}} & \frac{7 E_{\ell} I_{\ell}}{3 L_{i}}+\frac{G_{\ell} A_{f \ell} L_{i}}{36} & \frac{E_{\ell} I_{\ell}}{3 L_{i}}+\frac{G_{\ell} A_{f \ell} L_{i}}{36} \\
\frac{G_{\ell} A_{f \ell}}{L_{i}} & -\frac{G_{\ell} A_{f \ell}}{L_{i}} & \frac{E_{\ell} I_{\ell}}{3 L_{i}}+\frac{G_{\ell} A_{f \ell} L_{i}}{36} & \frac{7 E_{\ell} I_{\ell}}{3 L_{i}}+\frac{G_{\ell} A_{f \ell} L_{i}}{36}
\end{array}\right] \\
& \mathbf{b}_{12}^{(i \ell)}{ }^{T}=\left\{\frac{2}{3} G_{\ell} A_{f \ell}-\frac{2}{3} G_{\ell} A_{f \ell}-\frac{8 E_{\ell} I_{\ell}}{3 L_{i}}+\frac{G_{\ell} A_{f \ell} L_{i}}{9}-\frac{8 E_{\ell} I_{\ell}}{3 L_{i}}+\frac{G_{\ell} A_{f \ell} L_{i}}{9}\right\} \\
& b_{22}^{(i \ell)}=\frac{16 E_{\ell} I_{\ell}}{3 L_{i}}+\frac{4 G_{\ell} A_{f \ell} L_{i}}{9}
\end{aligned}
$$

A further reduction of the total number of unknowns from 5 to 4 is achieved in the next step. The slope defined at the mid-point of the beam, $\phi_{x m}^{(\ell)}$, can be condensed out of the strain energy expression, Eq. (A12), by employing the static condensation procedure. In the absence of nodal forces at the midpoint of the element, the first variation of the strain energy with respect to $q_{x 2}^{(i \ell)}$ yields the equilibrium equation at the mid-point as

$$
\mathbf{b}_{12}^{(i \ell)^{T}} \mathbf{q}_{x 1}^{(i \ell)}+b_{22}^{(i \ell)} q_{x 2}^{(i \ell)}=0
$$

Solving for $q_{x 2}^{(i \ell)}$ (i.e., $\phi_{x m}^{(\ell)}$ ) in the above equation and substituting into Eq. (A12), the strain energy expression reduces to

$$
U_{x}^{(i \ell)}=\frac{1}{2} \mathbf{q}_{x}^{(i \ell)^{T}} \mathbf{b}^{(i \ell)} \mathbf{q}_{x}^{(i \ell)}
$$

in which $\mathbf{q}_{x}^{(i \ell)}$ is identical to $\mathbf{q}_{x 1}^{(i \ell)}$, and $\mathbf{b}^{(i \ell)}$ is defined as 
or

$$
\mathbf{b}^{(i \ell)}=\left[\begin{array}{llll}
b_{11}^{(i \ell)} & b_{12}^{(i \ell)} & b_{13}^{(i \ell)} & b_{14}^{(i \ell)} \\
b_{12}^{(i \ell)} & b_{22}^{(i \ell)} & b_{23}^{(i \ell)} & b_{24}^{(i \ell)} \\
b_{13}^{(i \ell)} & b_{23}^{(i \ell)} & b_{33}^{(i \ell)} & b_{34}^{(i \ell)} \\
b_{14}^{(i \ell)} & b_{24}^{(i \ell)} & b_{34}^{(i \ell)} & b_{44}^{(i \ell)}
\end{array}\right]
$$

$$
\mathbf{b}^{(i \ell)}=\left[\begin{array}{cccc}
12 b_{1}^{(i \ell)} & -12 b_{1}^{(i \ell)} & 6 b_{1}^{(i \ell)} L_{i} & 6 b_{1}^{(i \ell)} L_{i} \\
-12 b_{1}^{(i \ell)} & 12 b_{1}^{(i \ell)} & -6 b_{1}^{(i \ell)} L_{i} & -6 b_{1}^{(i \ell)} L_{i} \\
6 b_{1}^{(i \ell)} L_{i} & -6 b_{1}^{(i \ell)} L_{i} & 4 b_{2}^{(i \ell)} & -2 b_{3}^{(i \ell)} \\
6 b_{1}^{(i \ell)} L_{i} & -6 b_{1}^{(i \ell)} L_{i} & -2 b_{3}^{(i \ell)} & 4 b_{2}^{(i \ell)}
\end{array}\right]
$$

where

$$
\begin{gathered}
b_{1}^{(i \ell)}=\frac{A_{f \ell} E_{\ell} G_{\ell} I_{\ell}}{L_{i}\left(12 E_{\ell} I_{\ell}+A_{f \ell} G_{\ell} L_{i}^{2}\right)} \\
b_{2}^{(i \ell)}=\frac{4 E_{\ell} I_{\ell}\left(3 E_{\ell} I_{\ell}+A_{f \ell} G_{\ell} L_{i}^{2}\right)}{L_{i}\left(12 E_{\ell} I_{\ell}+A_{f \ell} G_{\ell} L_{i}^{2}\right)} \\
b_{3}^{(i \ell)}=\frac{E_{\ell} I_{\ell}\left(6 E_{\ell} I_{\ell}-A_{f \ell} G_{\ell} L_{i}^{2}\right)}{L_{i}\left(12 E_{\ell} I_{\ell}+A_{f \ell} G_{\ell} L_{i}^{2}\right)}
\end{gathered}
$$

Substituting from Eq. (15) into Eq. (A1), the strain energy of the $\ell^{\text {th }}$ bolt is expressed as

$$
U_{x}^{(\ell)}=\sum_{i=1}^{K} \frac{1}{2} \mathbf{q}_{x}^{(i \ell)^{T}} \mathbf{b}^{(i \ell)} \mathbf{q}_{x}^{(i \ell)}
$$

As mentioned previously, the presence of nuts prevents the bolt from rotating at the end points; thus, it resembles clamped-type boundary conditions, requiring that $\phi_{x 1}^{(\ell)}=0$ and $\phi_{x K}^{(\ell)}=0$. Invoking this condition into Eq. (A18), the strain energy expression is modified as

$$
U_{x}^{(\ell)}=\frac{1}{2} \mathbf{q}_{x}^{\prime(l \ell)^{T}} \mathbf{b}^{\prime(l \ell)} \mathbf{q}_{x}^{(1 \ell)}+\frac{1}{2} \sum_{i=2}^{K-2} \mathbf{q}_{x}^{(i \ell)^{T}} \mathbf{b}^{(i \ell)} \mathbf{q}_{x}^{(i \ell)}+\frac{1}{2} \mathbf{q}_{x}^{\prime((K-1) \ell)^{T}} \mathbf{b}^{\prime((K-1) \ell)} \mathbf{q}_{x}^{\prime((K-1) \ell)}
$$

where the vectors and matrices with a prime are defined as 


$$
\begin{aligned}
& \mathbf{q}_{x}^{(1 \ell)^{T}}=\left\{\Delta_{x 1}^{(\ell)}, \quad \Delta_{x 2}^{(\ell)}, \quad \phi_{x 2}^{(\ell)}\right\} \\
& \mathbf{q}_{x}^{\prime((K-1) \ell)^{T}}=\left\{\Delta_{x(K-1)}^{(\ell)}, \quad \Delta_{x K}^{(\ell)}, \quad \phi_{x(K-1)}^{(\ell)}\right\} \\
& \mathbf{b}^{(1 \ell)}=\left[\begin{array}{ll:l}
b_{11}^{(1 \ell)} & b_{12}^{(1 \ell)} & b_{14}^{(1 \ell)} \\
b_{12}^{(1 \ell)} & b_{22}^{(1 \ell)} & b_{24}^{(1 \ell)} \\
\hdashline b_{14}^{(1 \ell)} & b_{24}^{(1 \ell)} & b_{44}^{(1 \ell)}
\end{array}\right]
\end{aligned}
$$

and

$$
\mathbf{b}^{\prime((K-1) \ell)}=\left[\begin{array}{ll:l}
b_{11}^{((K-1) \ell)} & b_{12}^{((K-1) \ell)} & b_{13}^{((K-1) \ell)} \\
b_{12}^{((K-1) \ell)} & b_{22}^{((K-1) \ell)} & b_{23}^{((K-1) \ell)} \\
\hdashline b_{13}^{((K-1) \ell)} & b_{23}^{((K-1) \ell)} & b_{33}^{((K-1) \ell)}
\end{array}\right]
$$

The terms on the right-hand side of Eq. (A19) can be rearranged such that all the unknown deflection and rotation components and the coefficients of the element stiffness matrices are assembled in large vectors and matrices. Hence, the strain energy expression is compacted to

$$
U_{x}^{(\ell)}=\frac{1}{2}\left\{\begin{array}{c}
\mathbf{q}_{x \Delta}^{(\ell)} \\
\mathbf{q}_{x \phi}^{(\ell)}
\end{array}\right\}^{T}\left[\begin{array}{cc}
\mathbf{b}_{\Delta \Delta}^{(\ell)} & \mathbf{b}_{\Delta \phi}^{(\ell)} \\
\mathbf{b}_{\Delta \phi}^{(\ell)} & \mathbf{b}_{\phi \phi}^{(\ell)}
\end{array}\right]\left\{\begin{array}{c}
\mathbf{q}_{x \Delta}^{(\ell)} \\
\mathbf{q}_{x \phi}^{(\ell)}
\end{array}\right\}
$$

where

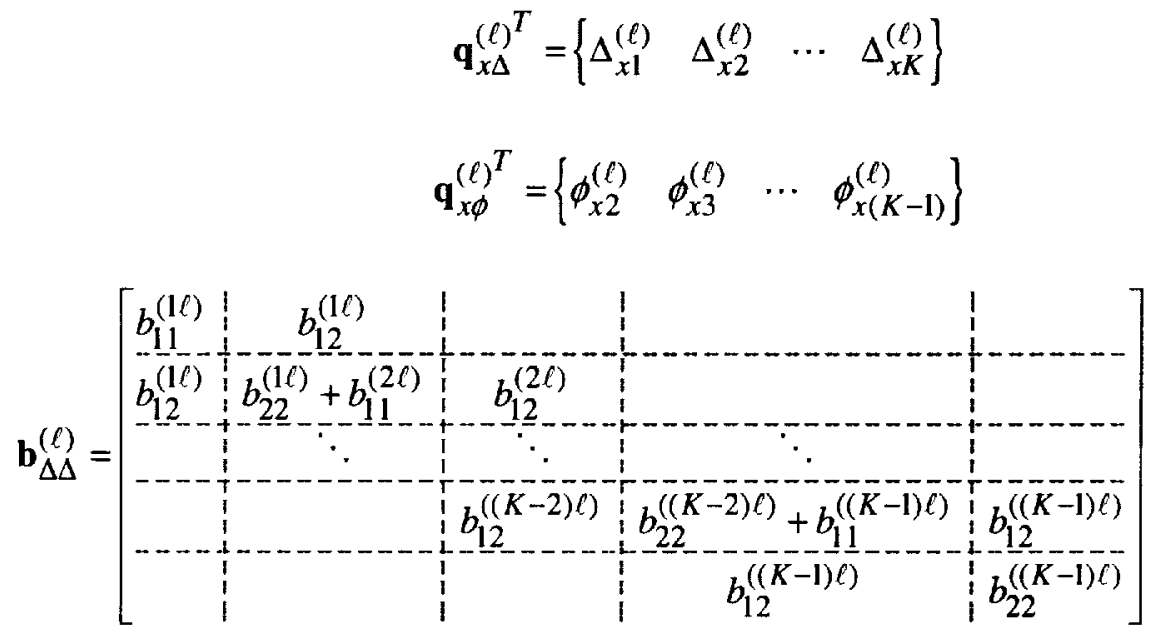




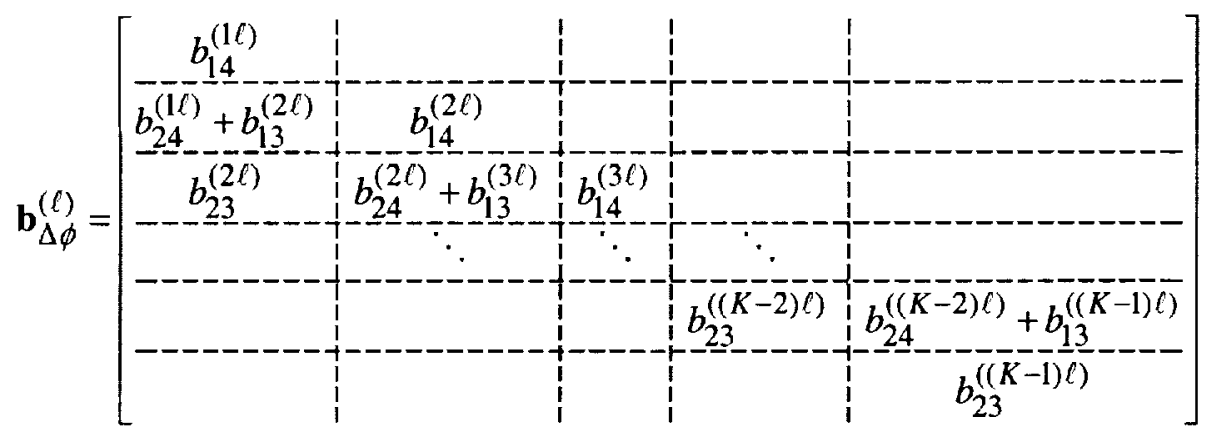

$(\mathrm{A} 22 \mathrm{c})$

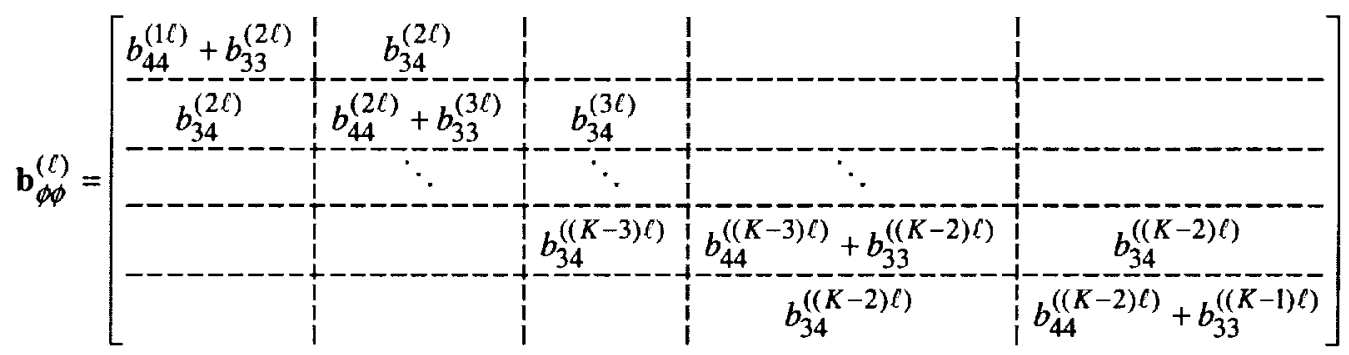

(A22d)

The bolt is subjected to contact forces that are assigned to the nodal points and the reaction moments due to clamped support at the end points of the bolt.

None of the internal nodal points, where the nodal rotations are active, is subjected to external moments. Therefore, it is appropriate to reduce the total number of unknowns by statically condensing out the rotational components of the bolt. Because no external moments are acting on the internal nodal points, the first variation of the strain energy with respect to the vector $\mathbf{q}_{x \phi}^{(\ell)}$ must vanish, thereby yielding the moment equilibrium equations in matrix form as

$$
\mathbf{b}_{\Delta \phi}^{(\ell)^{T}} \mathbf{q}_{x \Delta}^{(\ell)}+\mathbf{b}_{\phi \phi}^{(\ell)} \mathbf{q}_{x \phi}^{(\ell)}=\mathbf{0}
$$

Solving $\mathbf{q}_{x \phi}^{(\ell)}$ in the above equation and substitution into Eq. (A21), after rearranging the terms, lead to

$$
U_{x}^{(\ell)}=\frac{1}{2} \Delta_{x}^{(\ell)}{ }^{T} \mathbf{b}_{x}^{(\ell)} \Delta_{x}^{(\ell)}
$$

where the vector $\Delta_{x}^{(\ell)}$ is identical to $\mathbf{q}_{x \Delta}^{(\ell)}$ and the matrix $\mathbf{b}_{x}^{(\ell)}$ is defined as

$$
\mathbf{b}_{x}^{(\ell)}=\mathbf{b}_{\Delta \Delta}^{(\ell)}-\frac{1}{2} \mathbf{b}_{\Delta \phi}^{(\ell)} \mathbf{b}_{\phi \phi}^{(\ell)^{-1}} \mathbf{b}_{\Delta \phi}^{(\ell)}
$$

As mentioned previously, the stiffness properties of the bolt in the $(x, z)$ and $(y, z)$ planes are identical because the bolt material is isotropic and its cross-section is circular. 
Thus, the strain energy of the bolt in the $(y, z)$ plane can be expressed as

$$
U_{y}^{(\ell)}=\frac{1}{2} \Delta_{y}^{(\ell)}{ }^{T} \mathbf{b}_{y}^{(\ell)} \Delta_{y}^{(\ell)}
$$

in which the vector $\Delta_{y}^{(\ell)}$ contains

$$
\left.\Delta_{y}^{(\ell)}\right)^{T}=\left\{\begin{array}{llll}
\Delta_{y 1}^{(\ell)} & \Delta_{y 2}^{(\ell)} & \cdots & \Delta_{y K}^{(\ell)}
\end{array}\right\}
$$

and the matrix $\mathbf{b}_{y}^{(\ell)}$ is identical to $\mathbf{b}_{x}^{(\ell)}$, i.e.,

$$
\mathbf{b}_{y}^{(\ell)}=\mathbf{b}_{\Delta \Delta}^{(\ell)}-\frac{1}{2} \mathbf{b}_{\Delta \phi}^{(\ell)} \mathbf{b}_{\phi \phi}^{(\ell)^{-1}} \mathbf{b}_{\Delta \phi}^{(\ell)}
$$

The total strain energy of the bolt becomes

$$
U^{(\ell)}=\frac{1}{2} \Delta_{x}^{(\ell)^{T}} \mathbf{b}_{x}^{(\ell)} \Delta_{x}^{(\ell)}+\frac{1}{2} \Delta_{y}^{(\ell)^{T}} \mathbf{b}_{y}^{(\ell)} \Delta_{y}^{(\ell)}
$$

or, in a more compact form,

$$
U^{(\ell)}=\frac{1}{2}\left\{\begin{array}{c}
\boldsymbol{\Delta}_{x}^{(\ell)} \\
\boldsymbol{\Delta}_{y}^{(\ell)}
\end{array}\right\}^{T}\left[\begin{array}{cc}
\mathbf{b}_{x}^{(\ell)} & \mathbf{0} \\
\mathbf{0} & \mathbf{b}_{y}^{(\ell)}
\end{array}\right]\left\{\begin{array}{c}
\boldsymbol{\Delta}_{x}^{(\ell)} \\
\boldsymbol{\Delta}_{y}^{(\ell)}
\end{array}\right\}=\frac{1}{2} \mathbf{D}^{(\ell)} \mathbf{b}^{(\ell)} \mathbf{D}^{(\ell)}
$$

The analytical derivation of Eq. (A30) depends on the number of plates connected with the $\ell^{\text {th }}$ bolt. For example, the analytic derivation of matrix $\mathbf{b}_{x}^{(\ell)}\left(=\mathbf{b}_{y}^{(\ell)}\right)$ for bolts used in single- and double-lap joints is obtained as

with

$$
\begin{aligned}
& \mathbf{b}_{x}^{(\ell)}=\mathbf{b}_{y}^{(\ell)}=\left[\begin{array}{cc}
b^{*} & -b^{*} \\
-b^{*} & b^{*}
\end{array}\right] \\
& b^{*}=\frac{12 E_{\ell} I_{\ell} G_{\ell} A_{f \ell}}{L_{i}^{3} G_{\ell} A_{f \ell}+12 E_{\ell} I_{\ell} L_{i}}
\end{aligned}
$$

for a single-lap joint, and 


$$
\mathbf{b}_{x}^{(\ell)}=\mathbf{b}_{y}^{(\ell)}=\left[\begin{array}{c:c:c}
b_{11}^{(1 \ell)}-\frac{b_{41}^{(1 \ell)}}{b_{44}^{(1 \ell)}+b_{22}^{(2 \ell)}} & b_{13}^{(1 \ell)}-\frac{b_{14}^{(1 \ell)}\left(b_{34}^{(1 \ell)}+b_{12}^{(2 \ell)}\right)}{b_{44}^{(1 \ell)}+b_{22}^{(2 \ell)}} & -\frac{b_{14}^{(1 \ell)} b_{23}^{(2 \ell)}}{b_{44}^{(1 \ell)}+b_{22}^{(2 \ell)}} \\
\hdashline & b_{33}^{(1 \ell)}+b_{11}^{(2 \ell)}-\frac{\left(b_{34}^{(1 \ell)}+b_{12}^{(2 \ell)}\right)^{2}}{b_{44}^{(1 \ell)}+b_{22}^{(2 \ell)}} & b_{13}^{(1 \ell)}+\frac{\left(b_{34}^{(1 \ell)}+b_{12}^{(2 \ell)}\right) b_{23}^{(2 \ell)}}{b_{44}^{(1 \ell)}+b_{22}^{(2 \ell)}} \\
\hdashline \text { Sym. } & & b_{33}^{(2 \ell)}-\frac{b_{23}^{(2 \ell)^{2}}}{b_{44}^{(1 \ell)}+b_{22}^{(2 \ell)}}
\end{array}\right]
$$

with $b_{i j}^{(1 \ell)}$ and $b_{i j}^{(2 \ell)}$ defined by Eq. (A16) for a double-lap joint. If a bolt connects more than 3 laminates, the analytic derivation would be too lengthy to present herein. For this case, numerical calculation of the matrices becomes more appropriate.

\section{APPENDIX B}

The strain energy given as

$$
U^{(k)}=\frac{1}{2} \int_{A^{(k)}} N_{\alpha \beta}^{(k)} \varepsilon_{\alpha \beta}^{(k)} d A-\int_{A^{(k)}} N_{\alpha \beta}^{(k) *} \varepsilon_{\alpha \beta}^{(k)} d A
$$

can be rewritten in the form

$$
U^{(k)}=\frac{1}{2} \int_{A^{(k)}} \mathbf{N}^{(k)^{T}} \mathbf{\varepsilon}^{(k)} d A-\int_{A^{(k)}} \mathbf{N}^{(k)^{T} *} \varepsilon^{(k)} d A
$$

in which

$$
\begin{gathered}
\mathbf{N}^{(k)^{T}}=\left\{N_{x x}^{(k)}, N_{y y}^{(k)}, N_{x y}^{(k)}\right\} \\
\varepsilon^{(k)^{T}}=\left\{\varepsilon_{x x}^{(k)}, \varepsilon_{y y}^{(k)}, \varepsilon_{x y}^{(k)}\right\} \\
{ }^{*} \varepsilon^{(k)^{T}}=\left\{{ }^{*} \varepsilon_{x x}^{(k)},{ }^{*} \varepsilon_{y y}^{(k)},{ }^{*} \varepsilon_{x y}^{(k)}\right\}
\end{gathered}
$$

Substituting for the stress resultant vector, $\mathbf{N}^{(k)}$, given as

$$
\mathbf{N}^{(k)}=\mathbf{A}^{(k)} \boldsymbol{\varepsilon}^{(k)}
$$

leads to 


$$
U^{(k)}=\frac{1}{2} \int_{A^{(k)}} \varepsilon^{(k)^{T}} \mathbf{A}^{(k)} \varepsilon^{(k)} d A-\frac{1}{2} \int_{A^{(k)}} \varepsilon^{(k)^{T}} \mathbf{A}^{(k) *} \varepsilon^{(k)} d A \text { or }
$$

Its first variation becomes

$$
\delta U^{(k)}=\int_{A^{(k)}}\left\{\mathbf{A}^{(k)} \boldsymbol{\varepsilon}^{(k)}-\mathbf{A}^{(k) *} \boldsymbol{\varepsilon}^{(k)}\right\}^{T} \delta \boldsymbol{\varepsilon}^{(k)} d A
$$

Utilizing the kinematic relations

$$
\varepsilon_{\alpha \beta}^{(k)}=\frac{1}{2}\left(u_{\alpha, \beta}^{(k)}+u_{\beta, \alpha}^{(k)}\right)
$$

and invoking Eqs. (3) and (6) into Eq. (B5) result in

$$
\delta U^{(k)}=\int_{A^{(k)}}\left\{N_{\alpha \beta}^{(k)}-^{*} N_{\alpha \beta}^{(k)}\right\} \delta u_{\alpha, \beta}^{(k)} d A
$$

Before applying the divergence theorem, this expression can be rewritten as

$$
\delta U^{(k)}=\int_{A^{(k)}}\left[\left\{N_{\alpha \beta}^{(k)}-^{*} N_{\alpha \beta}^{(k)}\right\} \delta u_{\alpha}^{(k)}\right]_{, \beta} d A-\int_{A^{(k)}}\left\{N_{\alpha \beta, \beta}^{(k)}-^{*} N_{\alpha \beta, \beta}^{(k)}\right\} \delta u_{\alpha}^{(k)} d A
$$

Noting that ${ }^{*} N_{\alpha \beta, \beta}^{(k)}=0$ and applying the divergence theorem yield

$$
\delta U^{(k)}=\int_{\Gamma^{(k)}}\left\{N_{\alpha \beta}^{(k)}-{ }^{*} N_{\alpha \beta}^{(k)}\right\} n_{\beta} \delta u_{\alpha}^{(k)} d \Gamma-\int_{A^{(k)}} N_{\alpha \beta, \beta}^{(k)} \delta u_{\alpha}^{(k)} d A
$$

Finally, this expression can be recast as

$$
\begin{aligned}
& \delta U^{(k)}=-\int_{A^{(k)}} N_{\alpha \beta, \beta}^{(k)} \delta u_{\alpha}^{(k)} d A+\int_{\bar{\Gamma}^{(k)}}{ }^{*} t_{\alpha}^{(k)} \delta u_{\alpha}^{(k)} d \Gamma \\
& +\int_{\tilde{\Gamma}^{(k)}}^{*} t_{\rho}^{(k)} \delta u_{\rho}^{(k)} d \Gamma+\sum_{\ell=1}^{L} \int_{\hat{\Gamma}^{(k \ell)}}{ }^{*} t_{\rho}^{(k)} \delta u_{\rho}^{(k)} d \Gamma
\end{aligned}
$$

in which

with

$$
{ }^{*} t_{\alpha}^{(k)}=\left\{N_{\alpha \beta}^{(k)}-^{*} N_{\alpha \beta}^{(k)}\right\} n_{\beta} \text { and }{ }^{*} t_{\rho}^{(k)}=\left\{N_{\rho \eta}^{(k)}-{ }^{*} N_{\rho \eta}^{(k)}\right\} n_{\eta}
$$

$$
t_{\alpha}^{(k)} \delta u_{\alpha}^{(k)}=t_{x}^{(k)} \delta u_{x}^{(k)}+t_{y}^{(k)} \delta u_{y}^{(k)}=t_{n}^{(k)} \delta u_{n}^{(k)}+t_{s}^{(k)} \delta u_{s}^{(k)}=t_{\rho}^{(k)} \delta u_{\rho}^{(k)}
$$




\section{APPENDIX C}

As introduced by Lekhnitskii (1968), the displacement field, $u_{x}^{(k)}$ and $u_{y}^{(k)}$, and the in-plane stress resultants, $N_{x}^{(k)}, N_{y}^{(k)}$, and $N_{x y}^{(k)}$, satisfying the equilibrium equations and compatibility condition for the $k^{\text {th }}$ region (laminate) can be written in terms of arbitrary complex potential functions

$$
\left(u_{x}^{(k)}, u_{y}^{(k)}\right)=2 \operatorname{Re}\left\{\sum_{r=1}^{2}\left(p_{r k}, q_{r k}\right) \Phi_{r}^{(k)}\left(z_{r}^{(k)}\right)\right\}
$$

and

$$
\left(N_{x}^{(k)}, N_{y}^{(k)}, N_{x y}^{(k)}\right)=2 \operatorname{Re}\left\{\sum_{r=1}^{2}\left(\mu_{r k}^{2}, 1,-\mu_{r k}\right) \Phi_{r}^{(k)}\left(z_{r}^{(k)}\right)\right\}
$$

The complex constants, $p_{r k}$ and $\mathrm{q}_{r k}(r=1,2)$, are given by

$$
\begin{aligned}
& p_{r k}=a_{11}^{(k)} \mu_{r k}^{2}+a_{12}^{(k)}-a_{16}^{(k)} \mu_{r k} \\
& q_{r k}=a_{12}^{(k)} \mu_{r k}+a_{22}^{(k)} / \mu_{r k}-a_{26}^{(k)}
\end{aligned}
$$

in which the complex parameters $\mu_{1 k}$ and $\mu_{2 k}$ are the roots of the characteristic equation derived by Lekhnitskii (1968),

$$
a_{11}^{(k)} \mu_{r k}^{4}-2 a_{16}^{(k)} \mu_{r k}^{3}+\left(2 a_{12}^{(k)}+a_{66}^{(k)}\right) \mu_{r k}^{2}-2 a_{66}^{(k)} \mu_{r k}+a_{22}^{(k)}=0
$$

where $a_{i j}^{(k)}$, with $(i, j=1,2,6)$, are the compliance coefficients of the $k^{\text {th }}$ region (laminate).

For the $k^{\text {th }}$ region containing multiple bolt-holes, the complex potential functions, $\Phi_{r}^{(k)}$ and $\varphi_{r}^{(k)}$, can be expressed by superposing the complex potentials, $\Phi_{r}^{(k \ell)}$ and $\varphi_{r}^{(k \ell)}$, for individual bolt holes as

$$
\Phi_{r}^{(k)}\left(z_{r}^{(k)}\right)=\sum_{\ell=1}^{L^{(k)}} \Phi_{r}^{(k \ell)}\left(z_{r}^{(k \ell)}\right)
$$

and

$$
\varphi_{r}^{(k)}\left(z_{r}^{(k)}\right)=\sum_{\ell=1}^{L^{(k)}} \varphi_{r}^{(k \ell)}\left(z_{r}^{(k \ell)}\right)=\frac{d}{d z_{r}^{(k)}}\left[\Phi_{r}^{(k)}\left(z_{r}^{(k)}\right)\right]
$$

where $L^{(k)}$ is the number of bolt holes in the $k^{\text {th }}$ laminate and $\Phi_{r}^{(k \ell)}\left(z_{r}^{(k \ell)}\right)$ and $\varphi_{r}^{(k \ell)}\left(z_{r}^{(k \ell)}\right)$, with $r=1,2$, are the complex functions for the $k^{\text {th }}$ region containing the $\ell^{\text {th }}$ hole located at $\left(X_{\ell}, Y_{\ell}\right)$. The complex parameters $z_{r}^{(k)}$ and $z_{r}^{(k \ell)}$ are defined as 


$$
z_{r}^{(k)}=X+\mu_{r k} Y \text { and } z_{r}^{(k \ell)}=x^{(\ell)}+\mu_{r k} y^{(\ell)}
$$

in which $X$ and $Y$ are the global coordinates and $x^{(\ell)}$ and $y^{(\ell)}$ are the coordinates associated with the bolt hole. The explicit form of these complex potential functions can be expressed in the form

$$
\Phi_{r}^{(k \ell)}\left(z_{r}^{(k \ell)}\right)=\sum_{\substack{n=-N \\ n \neq 0}}^{N}\left(\xi_{r}^{(k \ell)}\right)^{n} \alpha_{r n}^{(k \ell)}
$$

and

$$
\varphi_{r}^{(k \ell)}\left(z_{r}^{(k \ell)}\right)=\sum_{\substack{n=-N \\ n \neq 0}}^{N} \frac{n}{\omega^{\prime}\left(\xi_{r}^{(k \ell)}\right)}\left[\left(\xi_{r}^{(k \ell)}\right)^{n} \alpha_{m}^{(k \ell)}\right]
$$

in which $\alpha_{r n}^{(k \ell)}$ are the complex unknown coefficients and $\xi_{r}^{(k \ell)}$ map the $\ell^{\text {th }}$ circular hole in the $k^{\text {th }}$ laminate to a unit circle in the mapped plane, thus permitting Laurent series representations to approximate the field variables. The prime denotes differentiation with respect to $\xi_{r}^{(k \ell)}$. The mapping functions $\xi_{r}^{(k \ell)}$ introduced by Bowie (1956) are in the form

$$
\xi_{r}^{(k \ell)}=\frac{z_{r}^{(k \ell)} \pm \sqrt{\left(z_{r}^{(k \ell)}\right)^{2}-a_{k \ell}^{2}\left(1-\mu_{r k}^{2}\right)}}{a_{k \ell}\left(1-i \mu_{r k}\right)}
$$

in which $a_{k \ell}$ is the radius of the $\ell^{\text {th }}$ hole in the $k^{\text {th }}$ region and $i=\sqrt{-1}$. The sign of the square-root term is chosen so that $\left|\xi_{r}^{(k \ell)}\right| \geq 1$. Inverting the mapping function provides

$$
z_{r}^{(k \ell)}=\omega\left(\xi_{r}^{(k \ell)}\right)=r_{r}^{(k \ell)} \xi_{r}^{(k \ell)}-\frac{s_{r}^{(k \ell)}}{\xi_{r}^{(k \ell)}}
$$

in which

$$
r_{r}^{(k \ell)}=\frac{a_{k \ell}}{2}\left(1-i \mu_{r k}\right), \quad s_{r}^{(k \ell)}=\frac{a_{k \ell}}{2}\left(1+i \mu_{r k}\right)
$$

The displacement components, $u_{x}^{(k)}$ and $u_{y}^{(k)}$, can be rewritten in terms of real vector quantities as

$$
\left(u_{x}^{(k)}, u_{y}^{(k)}\right)=\sum_{\ell=1}^{\ell^{(k)}} \sum_{r=1}^{2} \sum_{n=-N}^{N}\left(\mathbf{U}_{x(r n)}^{(k \ell)}{ }^{T}, \mathbf{U}_{y(r n)}^{(k \ell)}{ }^{T}\right) \mathbf{a}_{r n}^{(k \ell)}
$$




$$
\left(N_{x}^{(k)}, N_{y}^{(k)}, N_{x y}^{(k)}\right)=\sum_{k \ell=1}^{L^{(k)}} \sum_{r=1}^{2} \sum_{n=-N}^{N}\left(\mathbf{S}_{x x(r n)}^{(k \ell)}{ }^{T}, \mathbf{S}_{y y(r n)}^{(k \ell)}{ }^{T}, \mathbf{S}_{x y(r n)}^{(k \ell)}{ }^{T}\right) \mathbf{a}_{r n}^{(k \ell)}
$$

where the real vectors are given by

$$
\begin{aligned}
& \mathbf{U}_{x(r n)}^{(k \ell)}{ }^{T}=\left\{2 \operatorname{Re}\left[p_{r k}{ }^{(k \ell)} \Phi_{r n}^{*}\right],-2 \operatorname{Im}\left[p_{r k}{ }^{(k \ell)} \Phi_{r n}^{*}\right]\right\} \\
& \mathbf{U}_{y(r n)}^{(k \ell)}{ }^{T}=\left\{2 \operatorname{Re}\left[q_{r k}{ }^{(k \ell)} \Phi_{r n}^{*}\right],-2 \operatorname{Im}\left[q_{r k}{ }^{(k \ell)} \Phi_{r n}^{*}\right]\right\} \\
& \mathbf{S}_{x x(r n)}^{(k \ell)}{ }^{T}=\left\{2 \operatorname{Re}\left[\begin{array}{ll}
\mu_{r k}^{2} & { }^{(k \ell)} \varphi_{r n}^{*}
\end{array}\right],-2 \operatorname{Im}\left[\begin{array}{ll}
\mu_{r k}^{2} & { }^{(k \ell)} \varphi_{r n}^{*}
\end{array}\right]\right\} \\
& \mathbf{S}_{y y(r n)}^{(k \ell)}{ }^{T}=\left\{2 \operatorname{Re}\left[-^{(k \ell)} \varphi_{r n}^{*}\right],-2 \operatorname{Im}\left[-^{(k \ell)} \varphi_{r n}^{*}\right]\right\} \\
& \mathbf{S}_{x y(r n)}^{(k \ell)}{ }^{T}=\left\{2 \operatorname{Re}\left[\begin{array}{ll}
\mu_{r k} & { }^{(k \ell)} \varphi_{r n}^{*}
\end{array}\right],-2 \operatorname{Im}\left[\begin{array}{ll}
\mu_{r k} & { }^{(k \ell)} \varphi_{r n}^{*}
\end{array}\right]\right\} \\
& \mathbf{a}_{r n}^{(k \ell)^{T}}=\left\{2 \operatorname{Re}\left[{ }^{(k \ell)} \alpha_{r n}\right],-2 \operatorname{Im}\left[{ }^{(k \ell)} \alpha_{m}\right]\right\}
\end{aligned}
$$

The functions $\Phi_{m}^{(k \ell) *}$ and $\varphi_{r n}^{(k \ell)^{*}}$ are defined as

and

$$
\Phi_{r n}^{(k \ell)^{*}}\left(\xi_{r}^{(k \ell)}\right)=\left(\xi_{r}^{(k \ell)}\right)^{n}
$$

$$
\varphi_{r n}^{(k \ell)^{*}}=\frac{d}{d z_{r}^{(k \ell)}}\left\{\Phi_{r n}^{(k \ell)^{*}}\right\}=\frac{n\left(\xi_{r}^{(k \ell)}\right)^{n-1}}{\omega_{r}^{(k \ell)}}
$$

with

$$
\omega_{r}^{(k \ell)}=r_{r}^{(k \ell)}+\frac{s_{r}^{(k \ell)}}{\left(\xi_{r}^{(k \ell)}\right)^{2}}
$$

Terms arising from the expansion of $\mathbf{U}_{\alpha(m)}^{(k \ell)}, \mathbf{S}_{\alpha \beta(m)}^{(k \ell)}$, and $\mathbf{a}_{m n}^{(k \ell)}$ for $r$ ranging from 1 to 2 can be contained in the following vectors:

$$
\mathbf{U}_{\alpha(n)}^{(k \ell)^{T}}=\left\{\mathbf{U}_{\alpha(\ln )}^{(k \ell)^{T}}, \mathbf{U}_{\alpha(2 n)}^{(k \ell)}{ }^{T}\right\}
$$




$$
\begin{gathered}
\mathbf{S}_{\alpha \beta(n)}^{(k \ell)}{ }^{T}=\left\{\mathbf{S}_{\alpha \beta(1 n)}^{(k \ell)}{ }^{T}, \mathbf{S}_{\alpha \beta(2 n)}^{(k \ell)}{ }^{T}\right\} \\
\mathbf{a}_{n}^{(k \ell)^{T}}=\left\{\mathbf{a}_{1 n}^{(k \ell)^{T}}, \mathbf{a}_{2 n}^{(k \ell)^{T}}\right\}
\end{gathered}
$$

The terms arising from the expansion of these vectors for $n$ ranging from $-N$ to $N$ are contained in the vectors defined as

$$
\begin{gathered}
\mathbf{U}_{\alpha}^{(k \ell)^{T}}=\left\{\mathbf{U}_{\alpha(-N)}^{(k \ell)}{ }^{T}, \mathbf{U}_{\alpha(-N+1)}^{(k \ell)}{ }^{T}, \ldots, \mathbf{U}_{\alpha(N)}^{(k \ell)^{T}}\right\} \\
\mathbf{S}_{\alpha \beta}^{(k \ell)^{T}}=\left\{\mathbf{S}_{\alpha \beta(-N)}^{(k \ell)}{ }^{T}, \mathbf{S}_{\alpha \beta(-N+1)}^{(k \ell)}{ }^{T}, \ldots, \mathbf{S}_{\alpha \beta(N)}^{(k \ell)}{ }^{T}\right\} \\
\mathbf{a}^{(k \ell)^{T}}=\left\{\mathbf{a}_{(-N)}^{(k \ell)}{ }^{T}, \mathbf{a}_{(-N+1)}^{(k \ell)}{ }^{T}, \ldots, \mathbf{a}_{(N)}^{(k \ell)}\right\}
\end{gathered}
$$

with $(\alpha, \beta=x, y)$. Thus, using Eqs. (C24)-(C25), the series expansions for displacements and resultant stresses in Eqs. (C13) and (C14), respectively, can be rewritten as

$$
\begin{aligned}
& u_{\alpha}^{(k)}=\sum_{\ell=1}^{L^{(k)}} \mathbf{U}_{\alpha}^{(k \ell)^{T}} \mathbf{a}^{(k \ell)} \\
& N_{\alpha \beta}^{(\ell)}=\sum_{\ell=1}^{L^{(k)}} \mathbf{S}_{\alpha \beta}^{(k \ell)^{T}} \mathbf{a}^{(k \ell)}
\end{aligned}
$$

with $(\alpha=x, y)$. These expressions can be recast as

$$
\begin{aligned}
\mathbf{u}^{(k)} & =\sum_{\ell=1}^{L^{(k)}} \mathbf{U}^{(k \ell)^{T}} \mathbf{a}^{(k \ell)} \\
\mathbf{S}^{(k)} & =\sum_{\ell=1}^{L^{(i)}} \mathbf{S}^{(k \ell)^{T}} \mathbf{a}^{(k \ell)}
\end{aligned}
$$

by defining the following vectors

$$
\begin{aligned}
\mathbf{u}^{(k)^{T}} & =\left\{u_{x}^{(k)}, u_{y}^{(k)}\right\} \\
\mathbf{N}^{(k)} & =\left\{N_{x x}^{(k)}, N_{y y}^{(k)}, N_{x y}^{(k)}\right\}
\end{aligned}
$$




$$
\begin{gathered}
\mathbf{U}^{(k \ell)}=\left[\begin{array}{ll}
\mathbf{U}_{x}^{(k \ell)} & \mathbf{U}_{y}^{(k \ell)}
\end{array}\right] \\
\mathbf{S}^{(k \ell)}=\left[\begin{array}{lll}
\mathbf{S}_{x x}^{(k \ell)} & \mathbf{S}_{y y}^{(k \ell)} & \mathbf{S}_{x y}^{(k \ell)}
\end{array}\right]
\end{gathered}
$$

Finally, these equations can be expressed in a more compact form as

$$
\begin{aligned}
& \mathbf{u}^{(k)}=\mathbf{U}^{(k)^{T}} \mathbf{a}^{(k)} \\
& \mathbf{N}^{(k)}=\mathbf{S}^{(k)^{T}} \mathbf{a}^{(k)}
\end{aligned}
$$

where

$$
\begin{aligned}
\mathbf{U}^{(k)^{T}} & =\left[\begin{array}{llll}
\mathbf{U}^{(k 1)^{T}} & \mathbf{U}^{(k 2)^{T}} & \ldots & \mathbf{U}^{\left(k L^{(k)}\right)^{T}}
\end{array}\right] \\
\mathbf{S}^{(k)^{T}} & =\left[\begin{array}{llll}
\mathbf{S}^{(k 1)^{T}} & \mathbf{S}^{(k 2)^{T}} & \ldots & \mathbf{S}^{\left(k L^{(k)}\right)^{T}}
\end{array}\right] \\
\mathbf{a}^{(k)^{T}} & =\left\{\mathbf{a}^{(k 1)^{T}, \mathbf{a}^{(k 2)^{T}}, \ldots, \mathbf{a}^{\left(k L^{(k)}\right)^{T}}}\right\}
\end{aligned}
$$

\section{APPENDIX D}

The iterative scheme for solving the system of algebraic equations given in Eq. (69) begins with the initial estimates of $\theta_{A}^{(0)}$ and $\theta_{B}^{(0)}$, shown in Fig. D1, defining the contact region. These angles are measured in the counterclockwise direction from the $x^{(\ell)}$ axis of the local coordinate system, $\left(x^{(\ell)}, y^{(\ell)}\right)$. The initial estimate in most cases does not represent the true contact region, for which the radial stresses are all compressive $\sigma_{r r}^{(k)}<0$ on $\hat{\Gamma}^{(k \ell)}$, and the fact that the start and the end points of the contact region have zero radial stresses, $\sigma_{r r}^{(k)}\left(\theta_{A}\right)=0$ and $\sigma_{r r}^{(k)}\left(\theta_{B}\right)=0$.

As shown in Fig. D1, three distinct cases exist, depending on whether the radial stress near the start angle, $\theta_{A}^{(0)}$, is larger or smaller than the true value of the start angle or equal to the true value, $\theta_{A}$, of the contact region, $\hat{\Gamma}^{(k \ell)}$. The initial guess of the starting angle, $\theta_{A}^{(0)}$, is smaller than its true value, $\theta_{A}$, if the compressive radial stresses change sign and become tensile near the start point of the contact region, $\theta_{A}^{(0)}$. The initial guess of the starting angle, $\theta_{A}^{(0)}$, is larger than its true value, $\theta_{A}$, if the 


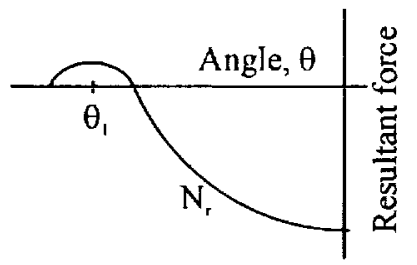

a)

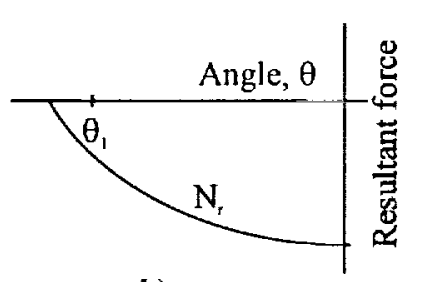

b)

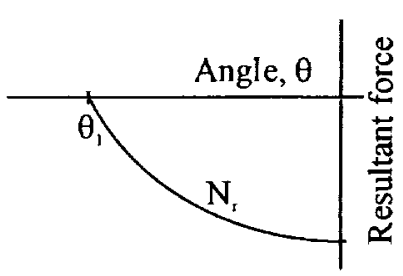

c)

Fig. D1. The behavior of radial stress near the start point of a contact region:

(a) the start angle is too small; (b) the start angle is too large;

(c) the start angle is correct.

compressive radial stresses do not change sign and remain compressive near the start point of the contact region. The initial estimate of the end angle, $\theta_{B}^{(0)}$, is larger than its true value, $\theta_{B}$, if the compressive stresses change sign and become tensile near the end angle. If the compressive stresses do not change sign, then the initial guess, $\theta_{B}^{(0)}$, is smaller than its true value.

During the iteration process, an initially guessed contact region defined by $\theta_{A}^{(0)}$ and $\theta_{B}^{(0)}$ converges to the true contact region defined by $\theta_{A}$ and $\theta_{B}$ through incrementally changing the values of the start and end angles. The increment is forced to decrease each time the true value of the start or end point is passed, and the direction of angle change is altered. The convergence of the iterative process is achieved when the incremental value of the angle reaches a pre-defined value.

In order to avoid the case of radial stresses having zero values at the start or end points of the contact region but with tensile and compressive stresses along the contact region, two auxiliary points are considered as shown in Fig. D2. These points are located inside the contact region near the start and end points of the contact region. In order to achieve convergence for the contact region, the radial stresses at these two auxiliary points must be compressive.

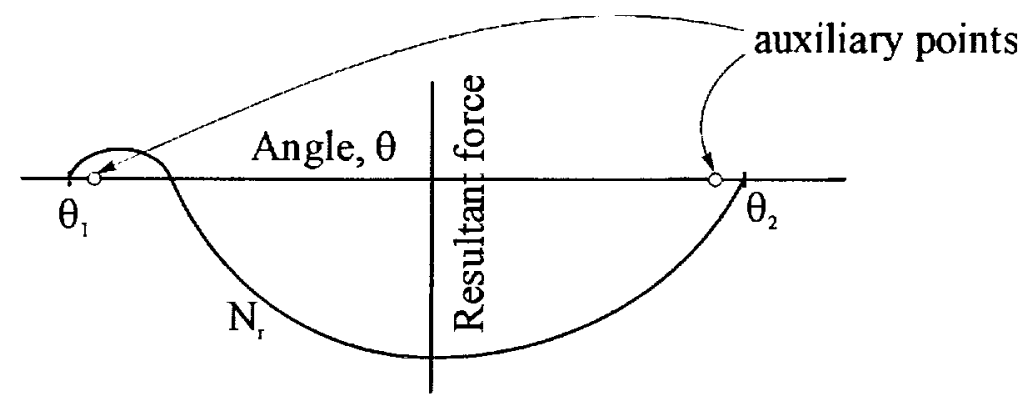

Fig. D2. Auxiliary points along a contact region. 


\section{USER: atila}

FILE: nasa.final.report.Aug

\section{END: atila@quartz+473.1}

